\title{
Civilisations
}

Revue internationale d'anthropologie et de sciences

humaines

40-1 | 1991

L'Asie et le Pacifique

\section{L'intégration du bassin pacifique : anticipations et fantasmes}

Eric Philippart

(2) OpenEdition

Journals

Édition électronique

URL : http://journals.openedition.org/civilisations/1687

DOI : 10.4000/civilisations. 1687

ISSN : 2032-0442

Éditeur

Institut de sociologie de l'Université Libre de Bruxelles

Édition imprimée

Date de publication : 1 janvier 1992

Pagination : 211-291

ISBN : 2-87263-063-5

ISSN : 0009-8140

Référence électronique

Eric Philippart, «L'intégration du bassin pacifique : anticipations et fantasmes », Civilisations [En ligne], 40-1 | 1991, mis en ligne le 07 juillet 2009, consulté le 01 mai 2019. URL : http://

journals.openedition.org/civilisations/1687; DOI : 10.4000/civilisations.1687

(c) Tous droits réservés 


\title{
L'INTEGRATION DU BASSIN PACIFIQUE : ANTICIPATIONS ET FANTASMES.
}

\author{
Eric PHILIPPART
}

- ô Pacifique souverain, mais où sont les vahinés d'antan ? -

\section{Première partie : Le Pacifique, "Nouveau Centre du Monde" - Les Théories Dominantes.}

Fin des années '80, impossible de lui échapper : le Pacifique est partout. Tout ouvrage traitant de l'évolution politicoéconomique de notre planète lui paie tribut.

Ouvrez, par exemple, le best-seller académique de l'historien Paul Kennedy "The rise and fall of great powers". Comme l'indique le sous-titre "economic change and military conflict from 1500 to 2000 ", cet ouvrage de près de 600 pages se consacre principalement à la recherche des grandes "lois" historiques de l'évolution des "balances of powers", avant de tenter une esquisse prospective des dix années à venir.

Après quelques considérations épistémologiques et méthodologiques "relativisantes" sur l'art de la prospective, $P$. Kennedy se limite à l'énoncé de deux tendances majeures déterminantes, selon lui, dans la problématique du jeu des puissances: "the rise of the Pacific region is likely to continue, simply because that development is so broad-based", commence-t-il prudemment pour enchaîner en citant sans réserves un "economic expert", selon lequel, "the center of the world economic gravity is shifting rapidly towards Asia and the Pacific"! [p. 441].

Cette évolution supposée ou affirmée, présente ou future est l'objet d'une littérature aussi abondante que variée dans sa provenance.

Une lecture superficielle de cette littérature peut donner 
l'impression que de nombreuses analyses de ce phénomène co-existent.

Mais lorsqu'on se penche sur les développements concrets qui suivent l'énoncé des concepts souvent vagues ou non définis, lorsqu'on passe du général au particulier, on constate qu'il existe un consensus assez large sur l'essentiel, c'est-à-dire sur la nature du phénomène, sur ses origines et sur ses limites géographiques.

Les variations portent en fait sur des questions d'ampleur et de tempo de cette évolution, sur son caractère désirable ou non, sur la pondération à donner aux différentes causes.

1.Nature du phénomène: des modulations nationales de fréquences!

Stuart Kirby dans "Towards the Pacific century" en fournit l'archétype, catégorie "enthousiaste/argumenté" [pp. 1-2] :

"This immense region holds most of the human and natural resources of the world - and in equal measure a major part of the prospects and hopes for economic development and for progress in general [...]. The modern school of "geopolitics" [...] pressed the view that the "heartland" of the world shifts in successive eras [...]; avowing that there has been the age of the Atlantic, the age of the Pacific will be the age of the future now impeding".

La majorité des auteurs se contentent de ces formules choc de basculement du centre du monde, du XXIe siècle - siècle du Pacifique, comme si cela était de toute évidence!

Plusieurs questions se posent pourtant.

En quoi consiste le centre du monde? Quelles sont ses relations avec la périphérie, càd le reste du monde? Quelles sont les relations en son sein? 
Des variations nationales assez nettes existent dans les réponses à ces questions.

Plus on appartient à un pays "développé" ou puissant de la région (USA, Japon, NPI,...), et potentiellement bénéficiaire, plus on semble vouloir nuancer la portée de ces hypothèses pour des raisons diverses (v. infra).

Plus on appartient à un pays dominé, petit ou exclus, et potentiellement perdant, plus on agite ces dernieres, généralement pour appeler à la fois à la vigilance et à la mobilisation face à cette évolution (URSS, ASEAN, Europe, ...).

\subsection{Les "puissants bénéficiaires".}

Ainsi, les écrits japonais traduits en anglais ou en français se sont caractérisés par un profil comparativement bas. La réalité de cette évolution ne fait aucun doute pour eux, mais ils restent mesurés dans leur analyse de son ampleur et de son caractère irréversible, et surtout du rôle futur dévolu au Japon. Censure ou auto-censure pour ne pas aggraver les problèmes d'image extérieure du Japon ? Perception plus aïgue de la fragilité du Japon induite par sa grande dépendance économique extérieure? Problème de gestion dialectique de deux stratégies déployées simultanément au niveau mondial et au niveau régional ? Tactique à court terme?

On trouve cependant des textes beaucoup plus explicites, voire brutaux, (peut-être liés au développement actuel du néo-nationalisme japonais) sur le type des relations externes et internes du nouveau centre du monde.

Les "Cahiers du Japon" ont ainsi publiés l'article d'un chercheur de l'Institut Nomura dont la teneur est la suivante [Eguchi Yujiro, p. 10].: 
"Lorsqu'ils tenaient ce rôle de principal créditeur, l'Angleterre puis les Etats-Unis avaient mis en place un système économique international dont ils assumaient la gestion. C'est maintenant au tour du Japon d'élaborer un ordre international qui lui convienne. [...]. Si, de part et d'autre du Pacifique, le Japon comme créditeur prépondérant et les Etats-Unis comme débiteur majeur, peuvent se compléter, il est possible d'envisager la formation de liens semblables à ceux qui, pardessus l'Atlantique, ont unil'Angleterre aux Etats-Unis pendant plus d'un siècle".

\subsection{Les "autres".}

Cette vision d'une alliance motrice de l'intégration, d'un condominium régional, est par contre très répandue chez les petits pays souvent méfiants et surtout chez les observateurs géographiquement extérieurs.

Ayant a priori plus à perdre qu'à y gagner, ils sont bien logiquement moins "frappés" par le sens exaltant de l'Histoire. Soit ils y voient le vieil impérialisme japonais ressuscité et métamorphosé, soit ils raisonnent en termes de coût/bénéfice et de realpolitik.

Ainsi, pour l'Institut du Pacifique (France), le capitalisme triomphant dépasse sa crise en déplaçant son centre dans le Pacifique où se met en place un condominium americanojaponais "fait davantage de rivalité feutrée que de confiance réciproque, d'intérêts bien compris que de partage de responsabilités". [J.P. Gomane et alii, p. 159].

Un ton au dessus, on retrouve cette vision dans les publications soviétiques autorisées.

Dans "La Vie Internationale", on peut lire [A. Bogomolov, p. 33], 
"c'est là (la région du Pacifique) que se trouve le centre de gravité des relations entre les deux principaux Etats du monde capitaliste: les USA et le Japon. [...]. Tous ces facteurs dans leur ensemble déterminent l'importance et l'influence internationales accrues de la région Asie-Pacifique. On est donc fondéà dire que les destinées $d u$ monde dépendront pour une large part $d u$ développement socio-économique et politique futur de cette région..."

L'auteur poursuit en dénonçant énergiquement la stratégie globale de l'impérialisme américano-nippon visant à isoler politiquement et économiquement les pays socialistes de cette évolution et de les empêcher de participer à la division du travail à l'échelle régionale !!

\subsection{Nature du phénomène.}

En définitive, si l'on tient compte de l'influence du contexte national sur la forme des théories, si l'on interprète raisonnablement leurs propos annexes, on peut résumer la problématique comme suit :

Il s'opère au profit du Pacifique un changement radical des rapports de force internationaux et inter-régionaux au niveau économique aujourd'hui, aux niveaux politique et stratégique demain.

Par centre du monde, on n'entend pas seulement un modele à suivre, un centre d'excellence ou un moteur de l'économie mondiale, mais l'endroit où les règles du jeu international seront décidées.

Il y a donc une notion de domination/dépendance dans ce concept centre/périphérie, les "extérieurs" étant de facto condamnés à devenir plus ou moins des filiales et des marchés secondaires.

Il est cependant entendu que cela ne signifierait pas nécessairement un impéralisme économique agressif, dans la 
mesure où les pays de la périphérie garderaient le choix : soit ne pas jouer le jeu, soit accepter ces règles fixées par le centre et au bénéfice du centre.

Les relations au sein du centre seraient marquées par un duumvirat Japon-USA, avec un rééquilibrage du rapport de force actuel au profit du Japon et division du travail précise entre eux.

2. Origines et indicateurs du phénomène : des analyses au diapason.

\subsection{Les indicateurs principaux.}

Les indicateurs sont bien sûr nombreux, mais il en est deux particulièrement sollicités, utilisés comme catalyseurs de la prise de conscience du "basculement" :

les taux de croissance des PNB et le volume d'échanges régionaux.

Remarquons sur ce point une confusion entre causes et conséquences d'autant plus largement répandue qu'on se trouve dans la catégorie fast food des théories.

Les forts taux de croissance économique sont fréquemment présentés comme étant à l'origine de l'émergence du Pacifique. Il s'agit, bien entendu, d'une explication tautologique par execellence, prenant une variable intermédiaire pour une cause.

2.2.Les origines.

Quant aux origines du phénomène, Frank B. Gibney, Président du Pacific Basin Institute (Santa Barbara -Californie), reprend les facteurs explicatifs généralement cités sous la 
forme de six dénominateurs communs de base ["The promise of the Pacific", BN/SR, feb. 1988, pp. 90-96] :

1) The modern advances in telecommunications and transportation have made the once huge oceanic distances shrink.

2) The strinkingly high rates of savings and investments.

3) The agricultural development, freeing more people and money for postwar industrialization.

4) The export-directed economies.

5) Free market economies led by consensus between the bureaucratic direction and the private sector.

6) The relative political stability.

La qualité des populations riveraines est un élément parfois ajouté à cette liste.

En résumé, cette croissance exceptionnelle des Etats du Pacifique proviendrait d'une structure différente et inédite de développement économique dans les rapports de production ( type de management, relations de travail, "capitalisme confucéen" ), dans les modes de production ( utilisation de nouvelles technologies, investissements massifs, ...), dans les types de production, etc.

Pour l'essentiel, ces explications prennent la forme de monographies largement descriptives, sans grand effort de conceptualisation, reposant sur des relations causales classiques (géopolitiques, économiques, démographiques,...).

Parmi les tentatives de théorisation/modélisation portant sur les origines du phénomène, la plus largement diffusée (à défaut d'être la plus solidement étayée) est celle notamment soutenue en France par J. Attali entre autres. Dans son livre "Les trois mondes, pour une théorie de l'après-crise" [Fayard, 
Paris, 1981], il se lance dans une démonstration historico-prospective de l'évolution des sociétés occidentales passant par les différents stades et localisations de "l'ordre marchand". De Bruges avec ses bateaux équipés de gouvernail d'étambot à Londres avec ses machines à vapeur, il énumère les centres successifs et les "breakthrough" technologiques associés à chaque stade de cet ordre marchand en terminant par le Pacifique et les technologies de l'information.

(Remarquons au passage qu'il ne précise pas la ville au coeur de ce nouveau centre. Lacune d'un modèle évolutionniste et historiciste, hâtivement construit, né d'une démarche nécessairement inductive, fonctionnant a posteriori et mal assuré dans ses projections du futur ? Prudence face à l'issue encore incertaine de la compétition entre Tokyo et Los Angeles?).

Ce type de théorie introduit dans ses démonstrations de nombreux concepts importés des sciences naturelles (thermodynamique, cybernétique, biologie organique,...) et les émaillent d'expressions choc forgées par les futurologues des années 60-70 (A. Toffler, H. Kahn,...), mélange de "troisième vogue" et de "société post-industrielle".

Il faut bien constater que, derrière l'idéalisation du système et du rôle des USA et/ou du Japon, ces auteurs renouvellent plus la forme que le fond du discours. Tous retombent dans le vieux rêve de la découverte des lois universelles et intemporelles du changement, dus sens de l'Histoire, de la succession linéaire et nécessaire des types de sociétés.

Certes le moteur du changement diffère (aujourd'hui, la majorité succomberait plutôt à la tentation monocausale technologiste) mais la logique est identique, or on connaît les multiples biais et faiblesses des typologies évolutionnistes. 
3. Limites géographiques du phénomène : de la grenouille au boeuf.

La terminologie pose ici problème, car nous nous trouvons face à des concepts équivoques ou non explicités, correspondant à des représentations mentales d'entités à la fois abstraites et de composition variable.

\subsection{La géographie classique.}

Du point de vue de la géographie classique, "le Pacifique" désigne conventionnellement la zone allant de l'Antarctique à l'Arctique, délimitée à l'ouest par l'Asie et l'Australie, à l'est par le continent américain. Cette zone de plus de 180 millions de $\mathrm{km} 2$ (un tiers de la surface de la planète) est un océan, c'est-à-dire qu'elle est occupée de façon quasi continue par de l'eau salée. Les seules terres émergées sont quelques guirlandes insulaires de taille très réduite (Mélanésie, Polynésie, Micronésie) occupées par une population très clairsemée et apathique selon les critères économistes occidentaux.

Du point de vue de la géographie humaine, de telles caractéristiques sont celles d'un obstacle naturel. Il s'agit, plus qu'autre chose, d'une non-entité, vide de terres et de populations humaines.

\subsection{Rim et contours.}

Comment les théoriciens du Pacifique peuvent-ils donc y voir le pôle de développement mondial présent et futur?

Ils pensent en fait à la périphérie, c'est-à-dire, aux pays riverains de cette zone et parlent $d^{\prime}$ ' un "bassin Pacifique" faisant de façon évidente, référence à la Méditerranée et à l'époque où celle-ci était le trait d'union d'une civilisation "avancée". 
Disons tout de suite que, sans même se pencher sur la question de l'homogénéité ou de la complémentarité de la périphérie pacifique (discutée infra), plusieurs différences physiques importantes existent entre ces deux cas et relativisent la validité d'un tel parallélisme.

Premièrement, l'échelle des distances est sans comparaison. On peut tenir ce fait pour secondaire en arguant du développement extraordinaire des communications et de la dématérialisation croissante de l'économie. Il existe néanmoins.

Deuxièmement, le bassin méditerranéen présente un continuum terrestre seulement interrompu par le détroit de Gibraltar, autrement dit sa périphérie correspond à la rive d'un grand lac. Le dit - bassin Pacifique, par contre, compte deux rivages complètement séparés et de façon très nette.

L' Encyclopedia Britannica parle d'ailleurs de "two majors physiographic regions, the Eastern and the Western Pacific", encore appelées "American Pacific" et "Asian Pacific".

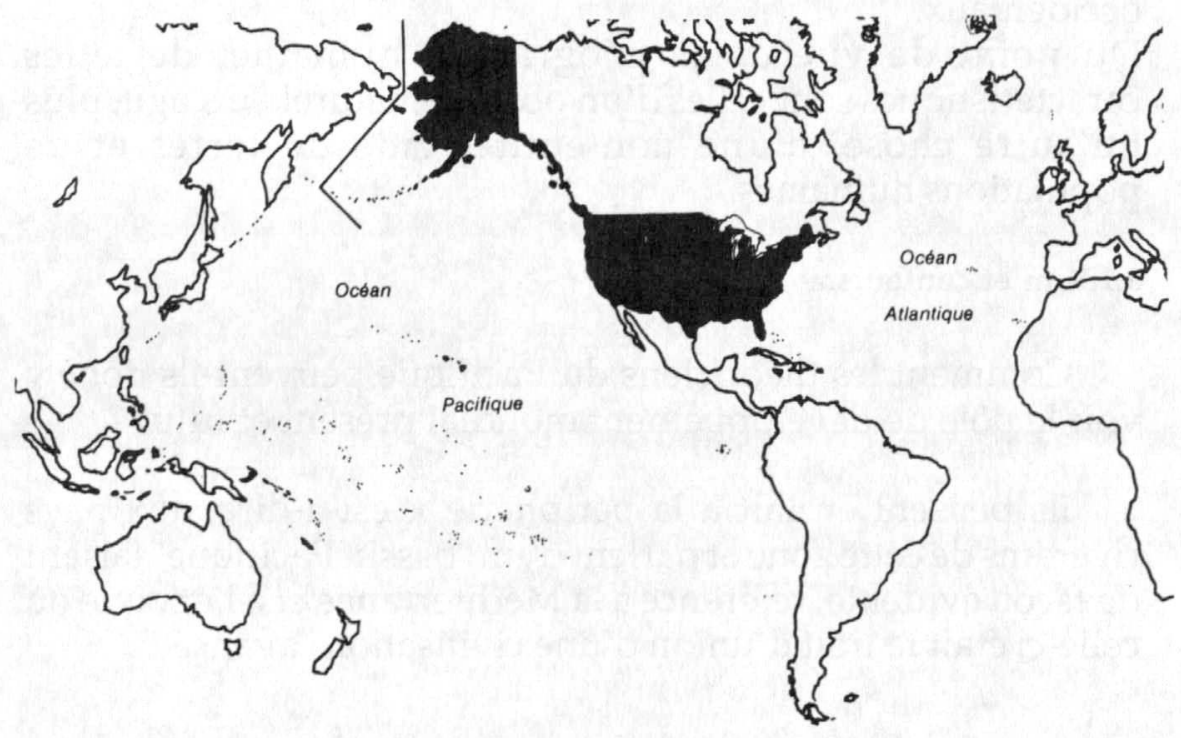


Un coup d'oeil à la carte ci-dessus (+ v. début du volume) et quelques ordres de grandeur sont assez éloquents :

En haut, nous trouvons la Sibérie face à l'Alaska, la péninsule du Kamtchatka face à la Colombie britannique. Ces territoires sont certes relativement proches les uns des autres mais ils ne sont quasiment pas peuplés (juste quelques centres de petite taille comme Anchorage, Vancouver, ...) et connaissent la majeure partie de l'année des conditions particulièrement peu propices aux communications (notamment Mers de Bering et d'Okhotsk gelées jusqu'aux archipels des Aléoutiennes et des Kourilles). Il faut donc considérer cette zone comme étant de facto une large coupure.

Nous trouvons ensuite le Japon, la péninsule coréenne et la côte chinoise jusqu'à Shangaï faisant face à la côte ouest des Etats-Unis. La plus petite distance trans-pacifique entre zones peuplées et économiquement actives se trouve à cette lattitude et dépasse les $8.000 \mathrm{~km}$ (par exemple Tokyo est plus près de Moscou ou du Koweit que de San Francisco !).

Plus bas, par ordre de proximité décroissante, Taiwan, les Philippines, le reste de la côte chinoise, la péninsule indochinoise et la Thailande sont les vis-à-vis du Mexique et des petits Etats d'Amérique centrale, séparés par des distances allant de 15.000 à plus de $18.000 \mathrm{~km}$ (c'est-à-dire à peu près des antipodes, l'un pour l'autre).

De part et d'autre de l'équateur, viennent alors la Malaisie, l'Indonésie et la Nouvelle-Guinée Papouasie en face de la Colombie et de l'Equateur, séparés en moyenne par $15.000 \mathrm{~km}$ d'océan.

Enfin nous avons la côte est de l'Australie et la Nouvelle Zélande en face du Pérou et du Chili, moins distants que les régions précédentes. Il faut cependant noter que le passage le plus court correspond à des conditions climatiques difficiles (les 40èmes rugissants) et relie deux zones relativement peu peuplées. 
Les caractéristiques topographiques d'un bassin ne sont donc pas réunies en l'espèce. Nous verrons plus loin que de fortes discontinuités existent également dans d'autres domaines.

\section{3."Morçeaux choisis".}

Le terme "bassin" doit être d'autant plus pris dans une acceptation sui generis non géographique, que, sous les titres flamboyants ("The age of the Pacific-Pacific Ocean turned into an inland sea" ou "the future lies in the Pacific", ...), l'écrasante majorité des auteurs ne parle soit explicitement que de la région Asie Pacifique, soit implicitement que d'une partie des pays riverains!

Dans le premier cas, on constate d'ailleurs souvent une exaltation, une emphase nationaliste asiatique qui ignore le conditionnel: "What is clear is the bright economic future of the region. That the 21st century will be the century of the Asia-Pacific is quite undisputable" [Prasert Chittiwatanapong, "Japan's role in the Asia-Pacific region", Korea and World Affairs, Autumn 88, p. 564].

Dans le second cas, le cadre de raisonnement adopté semble toujours ignorer ou exclure les pays communistes et les pays en voie de développement de la région (ce sont souvent les mêmes -URSS, Chine, Corée du Nord, péninsule indochinoise, Papouasie, Océanie -) ainsi que les pays d'Amérique centrale et d'Amérique latine.

Cet ensemble correspond en fait à la formule $5+6+x$ qui prévaut au sein du PECC -the Pacific Economic Cooperation Conference, v. infra-, à savoir les 5 "pays développés" (Australie, Canada, Etas-Unis, Japon, Nouvelle-Zélande), + pays membres de l'ASEAN (Association of South East Asian 
Nations/ Brunei, Indonésie, Malaisie, Philippines, Singapour, Thailande) + les NPI (Corée du Sud, Hong-Kong, Taiwan).

Le "Pacifique - centre du monde" est donc géographiquement parlant plutôt le Pacifique de l'hémisphère nord et plutôt le Pacifique asiatique. Economiquement parlant, il s'agit plutôt du Pacifique capitaliste, celui des "sociétés avancées", des Nouveaux Pays Industrialisés et des pays à revenus intermédiaires qui pour l'essentiel sont des économies de marché fortement impliquées dans l'économie mondiale.

4. Commentaire critique général des théories dominantes.

Un nombre de changements significatifs affectant le Pacifique se sont produits tant à l'intérieur qu'à l'extérieur de cette zone.

Comme nous le verrons dans la deuxième partie, ces changements sont d'ordre très divers (économiques, géopolitiques, ..., mais aussi psychologiques) et n'entretiennent pas toujours de rapports nécessaires entre eux. La combinaison, en partie fortuite, de ces éléments et leurs effets d'entraînement ont mis la question du Pacifique à l'ordre du jour, jusqu'à en créer un momentum "Pacifique" jamais vu dans l'histoire contemporaine.

Nous avons, par exemple, les phénomènes concommittants de l'émergence de l'Asie-Pacifique et la réorientation drastique des relations économiques extérieures de l'Australie et de la Nouvelle-Zélande vers le Pacifique notamment suite aux difficultés entraînées par l'intégration européenne.

Nous avons la haute visibilité médiatique de la vitalité californienne, "monde des gagneurs et du futur", et de son style 
de vie. On aurait très bien pû exprimer cette particularité en continuant à parler d'un contraste continental intra-US, côte ouest / côte est. Mais l'effet de mode et les changements susnommés aidant, on s'est mis à exprimer ce contraste en termes d'océans, en le transformant en clivage Pacifique / Atlantique.

L'exaltation des pays néophytes et le mécanisme psycho-sociologique de prédiction créatrice de réalité (par l'impact qu' elle a sur les actions des agents qui sont convaincus de sa véracité) ont vraisemblablement contribué au renforcement de l'image d'une "communauté" en gestation.

$* * * x+4$

La dimension Espace/Temps.

Les théories Pacifique dominantes, expression de ce momentum, ne sont pas de simples descriptifs de ces phénomènes.

Elles sont dans leur ensemble géographiquement et historiquement marquées, tant au niveau de la forme que du fond.

Espace :

Dans l'exposé qui est fait de la nature du phénomène, nous avons vu que des variantes nationales et régionales assez nettes

existent. Ces théories sont conditionnées par leurs lieux d'origine et de destination (conditionnement partiellement en fonction des intérêts locaux propres, des rapports entre l'entité nationale et l'ensemble Pacifique; plus commanditaires locaux; conditionnement en fonction de l'usage interne et/ou externe du discours).

Temps :

Ces théories sont, de plus, marquées de façon variable par des traits contemporains spécifiques.

En particulier, on peut se référer en l'espèce à trois d'entre eux: 
les traits épistémologiques dominants;

le statut de la science dans la société aujourd'hui et ses conséquences;

l'analyse actuelle des macro-tendances qui surdéterminent les relations internationales.

\subsection{L'épistémologie n'est plus ce qu'elle était.}

La science classique, parfois appelée newtonienne, se consacrait à l'identification des constantes, des séries causales récurrentes, ..., bref postulait que la norme était l'équilibre, le reste étant des phénomènes aberrants sans intérêt scientifique. $\mathrm{Au}$ cours de ce siècle, une révolution épistémologique renversant ce postulat a progressivement gagné toutes les disciplines scientifiques.

L'indéterminé, l'irréversible deviennent des concepts centraux. Les facteurs de discontinuité et de rupture retrouvent droit de citer dans l'élaboration des théories. Le changement devient l'axe en fonction duquel la recherche s'organise, jusqu'à en devenir un biais épistémologique important, c'est-à-dire que va se développer une tendance à chercher et à trouver systématiquement du changement radical partout, à surévaluer les indicateurs allant dans ce sens et à sous évaluer ou ignorer les autres.

Cette option épistémologique est, sans nul doute, une des causes de l'ampleur prise par le développement des théories "Pacifique". La pondération entre indicateurs ou l'absence de réserves à l'adresse d'indicateurs "confortants" va en ce sens.

\subsubsection{Examen des indicateurs}

Limitons nous à l'énoncé de quelques réserves dont pourraient être l'objet les deux principaux indicateurs 
unanimement avancés pour preuve du basculement du centre du monde : le volume des échanges transpacifique dépasse le volume des échanges transatlantique; les taux de croissance des PNB des pays du Bassin Pacifique sont très élevés et très supérieurs à ceux du reste du mode.

Concernant le premier de ces indicateurs, il y a bien sûr l'absence de définitions géographiques communes des entités concernées, les réserves d'usage concernant les méthodes de collecte des données et la variété de leurs sources

Le volume des échanges peut par ailleurs être biaisé par l'existence de différents phénomènes difficilement identifiables (dumping, simples mouvements comptables au sein d'entreprises transnationales, entre maison-mère et filiales, technique de délocalisation d'activités économiques, etc).

Toutes ces remarques rappellent simplement qu'il faut prendre ces chiffres pour ce qu'ils sont : des ordres de grandeur généraux plus ou moins fiables à la signification potentiellement multiple.

Il faut donc adopter une position de principe critique à l'égard des conclusions de domination économique qui sont tirées de cet indicateur. Il ne s'agit pas de dire que de telles conclusions ne peuvent être tirées. Nous disons à ce stade que pour qu'elles soient solidement étayées, elles doivent partir d'un indicateur beaucoup plus affiné (données plus désagrégées) et y adjoindre d'autres paramêtres.

Concernant le second de ces indicateurs, outre les considérations qui précèdent et toutes les réserves bien connues à l'égard de ce mode de mesure du développement économique (seul reflet de l'économie officelle, mode de calcul 
de l'inflation, le mode de redressement des données, etc), il est frappant de constater l'oubli des valeurs absolues.

Raisonner seulement en termes de taux et de pourcentage, est potentiellement trompeur.

Lorsque dans un processus de rattrappage économique, les positions initiales de chaque entité sont très éloignées, un différentiel de croissance ne débouche alors pas sur un changement "dramatique" des rapports de forces.

Dans les théories pro-Pacifique, le phénomène mis largement en exergue est celui de l'émergence rapide d'une partie de l'Asie-Pacifique où les économies connaissent des taux de croissance à deux chiffres, à savoir celles de l'ASEAN et des NPI.

Sont donc mises en avant d'une part des économies qui $n^{\prime}$ ont pas encore toutes maîtrisé leur problème démographique et/ou qui n'ont pas encore vraiment décollé, et d'autre part des économies ayant décollé mais dans des entités de taille et de poids économique moyennes ou petites.

En jouant à la bataille des chiffres avec les seules valeurs absolues, on pourrait fabriquer une toute autre image de la situation.

(Les Philippines, avec une population de 57 millions d'habitants en 1987, ont un PIB identique à celui de la Belgique, soit 86 milliards de $\$$. L'Indonésie, avec 170 millions d'habitants, a en 1987 un PIB inférieur à celui de la Belgique et des Pays-Bas additionnés -204 milliards de \$ internationaux contre 220 milliards-. La Thailande, avec 53 millions d'habitants, a un PIB de 122 milliards contre 134 milliards pour les Pays-Bas; etc. [chiffres extraits de Angus Maddison, "L'économie mondiale au 20e siècle", OCDE, Paris, 1989] ); (les quatre dragons -Taiwan, Corée du Sud, Hong-Kong, 
Singapour - totalisent une population d'un peu plus de 69 millions d'habitants, pour un PNB cumulé de 272 milliards de $\$$ en 1989 [source : the far East and Australasia 1987, 18th ed., p. 13], à peu près la moitié du PNB de l'Italie;

autre ordre de grandeur : en 1984, lorsqu'on additionne les PNB des quatre dragons et de l'ASEAN, on obtient pour une population d'environ 370 millions de personnes, un montant de $+/-491$ milliards de $\$$, alors que l'Australie, peuplée de 15 millions de personnes, a un PNB de 184 milliards de \$).

Aussi, en se placant à l'échelle mondiale, en soustrayant le poids du Japon et en caricaturant, les théories "Pacifique" reviendraient à soutenir que le centre du monde a basculé parce que s'est développée en quelques décades une puissance économique dont la taille actuelle avoisine celle de l'Italie et dont le retard du PNB par habitant par rapport à ceux de l'OCDE ne se résorbe pas ou lentement en dehors des NPI.

\subsubsection{Qualité des scenarii dérivés.}

Nos théories postulent implicitement des projections plus ou moins linéaires de ces taux de croissance à deux chiffres. Une telle certitude est peut-être péremptoire. En anticipant brièvement sur la deuxième partie, nous pouvons déjà faire différentes remarques.

L'histoire des économies industrialisées semble montrer que, depuis la 2ème guerre mondiale et jusqu'à présent, celles-ci connaissent une baisse tendancielle des taux de croissance, hors fluctuations conjoncturelles (moins forte chez les derniers arrivés que chez les premiers). Les taux très élevés de croissance sont caractéristiques des économies dans une phase de rattrappage; ils faiblissent avec les phases de maturité. 
En simplifiant, on comprend qu'il est plus facile en termes de mobilisation et d'allocation des ressources, d'augmenter production et productivité en partant d'un niveau faible que d'un niveau très elevé. Les pesanteurs structurelles changent avec le niveau de développement économique. Celles qui pèsent sur ce qu'on appelle les économies post-industrielles (où le tertiaire et le quaternaire sont largement dominants) sont les plus lourdes. Le Japon en fait aujourd'hui l'expérience (taux annuels moyens cumulés de croissance du PIB réel par habitant : $1950-1973=8 \% ; 1973-1987=2,8 \%$ - ce qui reste supérieur à la moyenne OCDE de 1,9\%)

Comme le dit A. Maddison, "à l'intérieur du groupe de l'OCDE, il y a eu convergence des revenus. Les pays les plus riches en 1900, $c^{\prime}$ est-d-direl'Australie, le Royaume-Uni, les Etats-Unis, les Pays-Bas et la Belgique ont accusé la croissance la plus lente de la production par habitant, alors qu'elle a été la plus rapide dans les pays les plus pauvres, c'est-à-dire le Japon, la Finlande et la Norvège" [A. Maddison, op. cit., p. 17 ].

Outre le fait de moindres pesanteurs structurelles, les pays suiveurs (en rattrappage de croissance) peuvent maintenir plus facilement des taux élevés d'augmentation de capital parce qu'ils n'ont pas à supporter les coûts de $R \& D$, la technologie étant copiée ou transférée grâce aux investissements étrangers.

Bref, hors Japon, le Pacifique dominateur, c'est soit plus petit soit moins impressionnant que ne le laissent penser les chiffres avancés.

En effet, dans la présentation et l'exploitation qui est faite des indicateurs du phénomène, il existe manifestement une surévaluation des uns et une sous-évaluation ou occultation des autres, qui est "conforme" à l'évolution épistémologique contemporaine.

(Scénarios pro-Pacifique à long terme basés sur des projections 
linéaires de facteurs de changement observés sur le moyen terme; passage sous silence des points de vulnérabilité qui affectent ce développement économique -v. 2ème partie).

\subsection{La science industrialisée}

\subsubsection{L'"outilisation" de la science.}

L'archétype du savant seul dans sa tour d'ivoire, détaché des contingences matérielles, à la recherche de la "vérité scientifique" comme d'un but en soi, a vécu. La révolution industrielle capitaliste a progressivement détruit le mythe porteur de la frugalité, de désintéressement, de la connaissance pour la connaissance. Le développement des capacités instrumentales de la technologie étant devenu le moteur de la production, les activités scientifiques ont été peu à peu intégrées dans la sphère économique et soumises à ses règles de fonctionnement (industrialisation, production de masse et impératifs de commercialisation de celle-ci). Les fonctions assignées aux différentes disciplines scientifiques diffèrent selon la nature de ces dernières.

La science est devenue un outil de production, mais aussi, bien plus qu'avant, un outil de gestion (expertise/aide à la décision) et de légitimation/conviction (argumentation pseudo-technocratique).

\subsubsection{Les conséquences.}

Les conséquences de cette évolution sont considérables tant sur le fond que sur la forme de la production académique issue des disciplines les plus en contact direct avec la sphère économique (qui est souvent aussi la plus largement diffusée). 
- Phénomène de mode.

Les disciplines qui se prêtent à une diffusion de masse (càd plutôt les sciences humaines) voient leur production de plus en plus ostensiblement traversée par des phénomènes de mode, dont les cycles semblent se raccourcir.

Le procédé de production s'opère par une réécriture du message de la théorie première, simplifié et standardisé. L'examen des données de base, la vérification sont des opérations longues et coûteuses. Rationalisation des coûts de production oblige : moins de démonstrations, plus d'affirmations.

- Commanditaires et médiatisation.

Qui dit vente dit acheteurs ou commanditaires.

Quels sont les artisans et les partisans de l'idée du Pacifique? A en juger par les seules réalisations concrètes à ce jour (v. infra), il s'agit presque exclusivement des grandes entreprises de la région et de quelques milieux académiques.

Pour des raisons historiques, ethniques, culturelles, ..., ce mouvement n'évoque, semble-t-il, pas grand chose parmi les différentes populations du Pacifique et manque donc cruellement de soutien populaire. Elle bénéficie au mieux d'une indifférence vaguement positive, l'idée suscitant parfois même des réactions nationalistes hostiles. Pour les mêmes raisons, les gouvernements concernés observent tous une prudente réserve, qu'elle soit tactique ou non (v. infra). Des hommes politiques et des hauts fonctionnaires participent bien aux travaux de fora et "colloques institutionnalisés", mais à titre privé.

Il s'agit donc d'emporter la conviction des opinions publiques et de la majorité des décideurs politiques. Des 
instituts travaillant à la carte et les "experts" mercenaires peuvent fleurir. Dans le cas des théories "Pacifique", cet aspect des choses s'est surtout développé aux Etats-Unis. Cette pratique y est-elle simplement plus affichée donc plus visible ou cela tient-il à des caractéristiques propres à cette société ? (on peut penser à plusieurs éléments explicatifs: mode de financement de la recherche; type de relations université/ secteur privé/Etat; tradition déjà ancienne de lobbying officiellement organisé; médiatisation très poussée du processus de décision politique; etc. ).

Médiatisation et discours académique. Comment convaincre?

Au niveau de l'"opinion publique", emporter la conviction suppose une médiatisation du projet. Or, pour accrocher, les medias à grande diffusion fonctionnent à la surenchère, au sensationalisme, aux formules choc à la façon des nouveaux philosophes, au radicalement nouveau, etc.

( Remarquons en passant qu'une telle prétention "révolutionnaire" en ce qui concerne le Pacifique est très surfaite. Des théories assez semblables avaient déjà fait souche dans les années vingt, où des auteurs comme Demangeon en France, annonçaient déjà le basculement du centre du monde dans le Pacifique, tandis que les hommes d'affaires "comptaient les têtes" en Chine en attendant le réveil de cette dernière).

Les nuances ne font pas bon ménage avec la forme médiatique. L'absence de conclusions concises et tranchées fait baisser l'indice d'écoute.

"Nous sommes au carrefour et nous entrons dans une nouvelle civilisation -après le Big Bang, le néolithique, la révolution industrielle, il y a maintenant, nous, qui créons 
et vivons "LE grand moment" (ce qu'on nous répète d'ailleurs tous les 5 ans depuis au moins 50 ans !). Voilà le langage qui accroche. La forme devient journalistique et la démonstration superflue.

Il y a une tentative de dramatiser les enjeux en fonction des situations respectives pour amener une mobilisation (que ce soit pour accéder au paradis promis ou pour préparer la contre-attaque).

Au niveau des décideurs, en pratiquant pour le surplus un lobbying plus "subtil", avec légitimation scientifique et démonstration sur mesure par "instituts" de circonstances et groupe d'études interposés, avec préparation de dossiers pour parlementaires, ministres et administration.

\subsection{3."Tous des vendus ?"}

Les lignes qui précèdent peuvent prêter à malentendu. Elles pourraient sembler décréter que tout auteur "pro-Pacifique" vend un salmigondis inventé de toutes pièces et est à la solde des transnationales américaines ou japonaises, pourrait-on dire.

Il ne $s^{\prime}$ agit bien entendu pas de faire ici de "terrorisme intellectuel". Soutenir que le bassin Pacifique est devenu ou deviendra le centre du monde est une analyse parfaitement défendable, plusieurs éléments allant en ce sens peuvent être avançés.

Notre évaluation des théories Pacifique doit être comprise de la façon suivante:

La littérature consacrée au Pacifique a connu un développement spectaculaire lors des années ' 80 . 
La partie dominante de cette littérature en termes de quantité et de visibilité (médiatisation) véhicule un cliché "engagé" et assez catégorique décrit en introduction qui est parvenu à devenir une évidence, un lieu commun grâce à la mise en avant de faits corroborants mais aussi suite a l'utilisation par certains de démonstrations biaisées (concepts élastiques, surpondération de certains éléments et/ou oubli d'autres données,...).

Cette utilisation s'est progressivement généralisée. Bien qu'il soit toujours difficile de distinguer ce qui a initié de ce qui a amplifié, nous soutenons simplement que les origines de cette multiplication ne sont pas seulement l'aveuglement nationaliste se transposant au niveau pacifique, le fastfoodisme intellectuel avec mode et conformisme, le manque d'extériorité ou l'auto-censure des auteurs, etc.. Nous soutenons qu'il y a une raison concommittante et peut-être supérieure à cela, à savoir l'existence d'enjeux concrets et l'industrialisation de la science.

Un tel développement quantitatif de production plus ou moins académique n'aurait, par exemple, pas été concevable si le champ d'investigation envisagé avait été l'identité du masque de fer parce que les enjeux sont différents.

Cependant, l'existence d'enjeux est en elle-même une condition nécessaire mais non suffisante pour entrainer un développement massif d'une production académique. Les parties prenantes à ces enjeux peuvent en effet poursuivre leur stratégie par d'autres moyens (distribution d'avantages matériels, intimidation, etc..). Penser utiliser la "science" à ces fins est un fait historiquement assez récent. Il suppose aussi qu'existe une "disponibilité" académique en ce sens.

Une fois encore, il ne s'agit donc pas de faire des procès d'intention ni de dresser des listes d'accusés de malhonnêteté 
intellectuelle, qui ne font en rien avancer l'analyse. Il s'agit de dire que parmi les écrits pro-pacifiques dont la démonstration est "expédiée", une partie importante n'a pas pour finalité première l'étude et la compréhension d'un phénomène, mais est d'abord une opération publicitaire de relations publiques, plus ou moins directement commanditée.

Ce fait est la conséquence de la nature du problème étudié combinée aux spécificités espace/temps occidentale et contemporaine dans les rapports science-société.

\subsection{Nature des paradigmes dominants les relations internationales.}

Par "paradigme", nous entendons le concept qui s'attache au nom de Thomas S. Kuhn. Dans son livre "The structure of scientific revolutions" [University of Chicago Press, 1962], il soutient que la connaissance évolue au travers d'une succession de paradigmes dominants. Cette approche est une approche épistémologique parmi d'autres. Beaucoup l'ont adoptée parce qu'ils en trouvaient des confirmations empiriques ou parce qu'ils étaient convaincus de sa puissance heuristique.

Les recherches épistémologiques portant sur la science des relations internationales et suivant le schéma "khunien", distinguent classiquement trois systèmes d'explication concurrents sur lesquels se construisent les théories contemporaines [v. K.J. Holsti, "The Dividing discipline. Hegemony and diversity in International theory", Allen and Unwin, Boston, 1985, 165 p.] :

le paradigme classique où l'élément principal est l'Etat;

le paradigme de la "société globale" où l'élément principal est l'interdépendance croissante créatrice de besoins fonctionnels nouveaux;

le paradigme marxiste ou "dépendantiste" avec la transposition 
de l'exploitation de classes à l'échellon international (échange inégal et impérialisme structurel).

Les rapports de forces existant aujourd'hui entre ces paradigmes semblent être les suivants :

le paradigme classique autrefois écrasant connaît une démonétisation relative;

le paradigme marxiste également, mais apparemment plus par contrecoup de l'évolution politique que par ses faiblesses intrinsèques;

reste donc le paradigme de la "société globale" qui n'a pu que gagner en importance.

\subsubsection{Les options du paradigme de "la société globale".}

Exigence fonctionnelle du multilatéral.

Le credo de ce dernier basé sur le défi du "stretching of governance", l'inadaptation de la taille des Etats par rapport à l'échelle désormais globale des problèmes - environnement, économie, etc. -, est aujourd'hui devenu une sorte de litanie obligée pour tout discours officiel.

Ce credo, postulant l'importance déterminante de ce que A. Giddens ["The constitution of society - outline of the theory of structuration", Polity Press, Cambridge, 1986] appelle la dimension "inter-sociétale", se focalise donc sur les phénomènes multilatéraux, les processus d'intégration régionale,..., qui sont tenus pour vitaux puisqu'ils remplissent le besoin fonctionnel de coordination et renforcent l'adaptabilité des systèmes à leur environnement. Ces phénomènes sont donc particulièrement valorisés puisqu'ils vont dans le "sens de l'Histoire".

Il n'est dès lors pas interdit de penser que les progrès d'un paradigme qui focalise ses investigations sur les phénomènes 
d'interdépendance et l'émergence de structures transcendant les nations aillent de pair avec la multiplication des auteurs ayant une propension à trouver de la cohérence supranationale, de la communauté transnationale, ..., là où elles ne sont pas nécessairement, ou ayant une propension à surpondérer certains éléments confortants l'évolution attendue a priori.

\subsubsection{Le primat de l'Economique.}

Plusieurs phénomènes historiques nouveaux des dernières décennies ont débouché sur une autre révision du cadre conceptuel appliqué à l'étude des relations internationales.

D'une part, les affrontements idéologiques, politiques ou militaires ont diminué de façon sensible au niveau international (achèvement des processus de décolonisation; fin de la guerre froide (beaucoup considèrent que l'Afghanistan ne fut qu'un accident ou une péripétie); progressif déclin idéologique des marxismes et prétention a-idéologique occidentale; désengagement progressif des super-puissances devant plus se concentrer sur leurs problèmes intérieurs principalement économiques, etc.)

$D^{\prime}$ autre part, et en partie par contrecoup, les aspects économiques ont pris une place grandissante dans les affaires internationales (mondialisation de l'économie; problème de la dette; etc.).

Restent les autres enjeux qui demeurent relativement mineurs sans doute de par leur évolution continue et lente, plus indolore parce que plus "insidieuse" : tant la dimension culturelle (poursuite de l'occidentalisation) que la dimension linguistique (domination quasi totale de l'anglais) ont perdu, 
à l'échelle de l'histoire contemporaine, de leur visibilité, voire de leur importance, dans les relations internationales.

La hiérarchie des facteurs déterminants des relations internationales s'est alignée sur cette évolution historique : un fort a priori s'est développé rétrogradant les diverses dimensions au profit de la dimension économique devenue la dimension la plus pregnante, la plus significative!

\section{En un mot ...}

Bref, lorsque vous étudiez les relations internationales au travers d'un cadre conceptuel général où le changement est la norme et $d^{\prime} u n$ cadre conceptuel particulier où la logique du transnational et de l'économique surdétermine les autres données, alors l'émergence d'une Communauté du Pacifique, le siècle du Pacifique, ..., vous sembleront beaucoup plus évidents qu'à tout autre, parce qu'ils correspondent à vos schémas mentaux antérieurs.

Or ces cadres sont aujourd'hui de plus en plus "populaires" au sein de la discipline des relations internationales. C'est en cela aussi que l'on peut dire que ces théories du Pacifique sont conditionnées par des spécificités contemporaines extérieures au problème du Pacifique proprement dit.

En conclusion de cette évaluation critique rapide, il faut donc garder un sens critique aigu à l'encontre des théories Pacifiques. Il faut enfin constater qu'il n'existe pas d'évidences tranchées sur le sujet. Nous allons nous attacher à développer une autre vision du phénomène Pacifique dans la partie qui suit. 
Deuxième partie : Les caractéristiques fondamentales de l'entité géographique "Pacifique", hier et aujourd'hui.

\section{Background Pacifique.}

Il n'est pas d'analyse du contemporain sans un minimum de recul historique.

Qu'en est-il de l'arrière-fond du phénomène Pacifique?

- Une première constante: cette zone était et reste hautement hétèrogène à tous les points de vue (ethnique /linguistique /culturel / religieux; systèmes politique /idéologique /social; niveau de développement économique).

- Une seconde constante : cette hétérogéneïté était et reste potentiellement explosive.

- Un premier changement : cette zone fut sans doute la zone de conflits la plus importante de l'après-deuxième guerre mondiale (guerres civiles; guerres de libération et de décolonisation, terrain d'affrontement des super-puissances, conflits régionaux "classiques").

Elle bénéficie à présent d'une normalisation et d'une stabilisation qui sont attribuées, quasi exclusivement et de façon abusive à notre avis, à la politique américaine dans la zone (la "pax americana").

a Un second changement : cette zone a connu des évolutions économiques très différenciées où l'Asie stagnait loin derrière les autres parties. Il y a aujourd'hui 
une inversion partielle de cette différenciation au profit de l'Asie.

2.Le bilan économique - Le centre du monde vu de la périphérie

\subsection{Aperçu historique.}

En étudiant la croissance économique du monde depuis le début du siècle, des études solides suggèrent les évolutions globales suivantes qui ne correspondent pas aux clichés bien en place :

Depuis 1900, le taux de croissance cumulé du "PIB" mondial est de $3 \%$ par an.

Le meilleur bilan est celui de l'Amérique latine (!) avec 4,1\% par an, puis celui de l'OCDE avec $3 \%$ et enfin l'Asie (incluant donc la Chine et l'Inde) avec 2,7\% l'an.

A l'échelle nationale, Taiwan et le Brésil viennent en tête avec $5,1 \%$ et $4,8 \%$ de croissance du PIB. Quand on considère la croissance du revenu par habitant, ce sont le Japon et Taiwan qui arrivent en tête avec $3,1 \%$ et $2,8 \%$ par an.

Le premier élément qui ressort des comparaisons globales est que la disparité des revenus par habitant s'est très fortement accrue entre les différentes zones.

(En 1900, l'écart de revenu par habitant entre la moyenne OCDE et la moyenne de l'Asie est de 5 à 1; en 1987, il est de 8 à 1). Le différentiel de croissance démographique explique cela.

Le second élément est l'accélération du rythme de croissance économique dans toutes les régions après 1950 (1900-1950= moyenne mondiale de 2,1 \% par an; 1950-1987 = 4,2 \%), mais plus particulièrement en Asie (où la croissance est huit fois plus rapide depuis 1950 qu'entre 1900-1950). 
Bref, il ressort de cette étude qu'en ce qui concerne le "bassin Pacifique des théories" (v. supra formule $6+5+X$ ), il y a, à l'échelle du siècle, des évolutions clairement marquées :

- celle, continue, des pays OCDE de la zone qui ont connu des changements structurels convergents et un resserrement des écarts de leurs revenus par habitant respectifs (les pays les plus riches en 1900 - USA, G-B, Belgique, ... - ayant la croissance la plus lente et les plus pauvres - Japon, Norvège, ... - la plus rapide);

- celle, plus chaotique, des autres pays qui, par comparaison, commencent tous par une très longue période de stagnation globalement suivie, durant les trente dernières années, par une phase d'accélération de la croissance.

Cette accélération ne se transforme en rattrappage que dans quelques pays qui, après 1950, ont réussi à initier une dynamique de croissance très supérieure (maîtrise de la croissance démographique; taux de création de capital très élevés; augmentation du rapport travail-population; effort dans l'enseignement; importation à faible coût de technologies occidentales; conjoncture économique internationale favorable et conditions privilégiées d'exportation - SPG sur le marché US ...).

\subsection{Les autres indicateurs du "Centre du monde"?}

On part généralement du postulat que l'influence sur l'économie mondiale se mesure par le volume et la qualité des échanges de services, de biens et de capitaux d'un pays avec le reste du monde. 
- En ce qui concerne le volet des échanges de biens et services, en s'en tenant aux données officielles avec les réserves que l'on sait, nous avons les ordres de grandeur suivants.

1986, montant d'exportations par an et par habitant :

Indonésie : 89 ;

Philippines : 87 ;

Thailande : 169 ;

Corée du Sud : 835 ;

Taiwan : 2.056 \$;

alors que la moyenne OCDE est de 3.411 (Japon : $1.735 \$$ ).

En valeur absolue, nous avons, en milliards de \$:

la RFA avec $248 \mathrm{M}$ \$;

les USA avec $193 \mathrm{M}$ \$;

le Japon avec $182 \mathrm{M}$ \$;

la G-B avec $136 \mathrm{M}$;

la France avec $129 \mathrm{M} \$$;

l'Italie avec $97 \mathrm{M} \$$;

l'URSS avec $92 \mathrm{M} \$$; ....;

Taiwan avec moins de $20 \mathrm{M}$ \$;

la Corée avec $9,4 \mathrm{M}$ \$; etc.

Le géant indonésien avec ses 162 Millions d'habitants vient très loin avec seulement $14,5 \mathrm{M}$ \$.

Si l'on s'en tient à un instantané de la situation actuelle sur base des chiffres qui précèdent, on obtient une image des rapports de force économiques internationaux très éloignée de celle proclamée par les théories du Pacifique.

Comme l'écrit A. Maddison ["L'économie mondiale au XXème siècle", p. 31], 
"dans la mesure où les influences de la croissance se transmettent par le biais du commerce mondial, il est clair que les pays de $l^{\prime} O C D E$ ont exercé une influence majeure durant tout le vingtième siècle (...) avec $73 \%$ des exportations mondiales en 1900 et $67 \%$ en 1986. Les exportations et leurs politiques commerciales ont elles ausi exercé une influence majeure sur le bilan de l'économie mondiale".

- En ce qui concerne le volet des mouvements de capitaux, les données disponibles sont plus parcellaires et plus difficiles à interpréter.

Néanmoins, là aussi, la ligne de partage est claire :

les clivages ne sont pas géographiques. Il y a simplement l'OCDE et le reste du monde. Au sein de l'OCDE, il y a l'irrésistible ascension du Japon (devenu gros exportateur de capitaux, mais aussi, ce qui n'est paradoxal qu'en apparence, premier emprunteur mondial sur les marchés internationaux en 1989).

Ceci n'est pas suffisant que pour déboucher sur une contre-théorie Pacifique, mais c'est assez que pour une fois encore en relativiser les affirmations.

3.Le modèle de croissance qui a changé le futur : bis repetitat non credet.

Le 21ème siècle sera-t-il le siècle du Pacifique?

Pour répondre à cette question, il faut au préalable rechercher les causes de l'indéniable dynamisme et réussite économiques dont font preuve plusieurs entités de la zone Pacifique (dynamisme que l'on étend abusivement à l'ensemble).

Autrement dit, existe-t-il un modèle de croissance particulier 
au Japon et aux NICs/NPI?

Ce modèle est-il transposable et est-il en cours de transposition?

Sa pérennité est-elle garantie?

L'explosion asiatique prend historiquement naissance à "l'âge d'or" (1950-1973) de l'économie mondiale. Le cas du Japon vient d'abord à l'esprit, bien que son développement spectaculaire avait déjà pris auparavant son envol.

Mais le modèle de croissance le plus rapide de tous est celui des Quatre dragons. C'est le plus traumatisant, celui qui frappe le plus les esprits et celui qui a produit l'image abusive du dynamisme extraordinaire de la région Pacifique dans son ensemble.

C'est donc sur ce modèle spécifique qu'il faut se pencher pour examiner les réalités nouvelles du Pacifique. La littérature consacrée aux NPI ou NICs asiatiques s'est, elle aussi, fort développée et les modèles d'explication ne manquent pas.

\subsection{Détermination "exogène majoritaire" :}

M.B. Yahuda, par exemple, explique le dynamisme du Pacifique de la façon suivante [Michael B. YAHUDA, "The Pacific Community - not yet", The Pacific Review, Oxford University Press, vol.1, n2, 1988 ].:

"It is abundantly clear that it is the United States above all that has paid the costs for providing the economic environment in which Japan and the NICs of East Asia have been able to flourish. In addition to the provision of a secure international environment, the United States extended capital and aid at crucial points in the development of these economics, it facilitated technology transfers, assisted in education and above all it opened the United States 
domestic markets to their produce without demanding reciprocal treatment for american produce in their markets"

Si l'on s'en tient strictement à cette explication, le modèle $n^{\prime}$ est pas transposable aux autres pays de la zone et est même menaçé dans sa pérennité.

Ce que les Etats-Unis pouvaient faire en termes de sécurité, d'aide, de transfert et d'ouverture économique au temps de leur apogée pour le Japon et les NICs, ils ne peuvent le faire aujourd'hui à l'échelle du Pacifique. Le relatif déclin économique et militaire américain rend peu vraisemblable le maintien de l'environnement stratégique et économique que les USA ont créé dans le Pacifique. Ils ne semblent de toutes façons pas ou plus y être disposés.

Dans cette hypothèse, des menaces planent sur la réussite future du Pacifique ou, tout au moins, des solutions alternatives solides doivent être rapidement trouvées. En effet, les Etats-Unis répètent avec de plus en plus d'insistance que les bénéfices qu'ils retirent leur action dans le Pacifique sont sans proportion avec les coûts et parlent d'un désengagement partiel (non défini). Cette insistance est aujourd'hui d'autant plus forte que les problèmes du déficit budgétaire US et de déséquilibre de sa balance des paiements deviennent très pressants.

Etant donné que l'autre super-puissance, l'URSS, est également à la recherche d'une "lower cost foreign policy", cela signifie en fonction des possibilités soit des pressions accrues sur les "seconds couteaux" pour le burden sharing, soit des réductions drastiques dans les engagements. L'URSS a déjà entamé un désengagement tous azimuts qui donne le vertige. Les problemes de vide de pouvoir dans la zone Pacifique ne peuvent être exclus. 
Indépendamment de ces problèmes ponctuels de burden sharing trans-pacifique, les priorités régionales d'une super-puissance sont par définition fluctuantes puisque sa stratégie est globale.

Il y a quelques années, en 1982, on pouvait par exemple lire dans un article consacré à l'Alliance atlantique publié par "The Economist" ces propos reflètant assez l'air du temps.:

"the Americans are finding ... Europe undynamic, muddlehead or unreliable in comparison with the realistic and striving Japanese and others. (...). There is therefore a not inconsiderable possibility of impatient Americans saying 'the hell with Europe; but not so much to retreat to a fortress America as to turn from the Atlantic to the the Pacific as their principal external alliance".

Depuis, il y a eu le lancement du projet "Europe 1992" par la Communauté européenne, l'arrivée des réformateurs en URSS et la chute du rideau de fer...

L'Association américaine des Européanistes, morte de sa belle mort, renait de ses cendres. L'Europe est revenue au top de l'agenda du Président Bush.

$\mathrm{Ce}$ dernier exemple montre combien la prospective est une discipline hasardeuse. Cela vaut tout particulièrement pour le Pacifique.

3.2.Détermination "endogène majoritaire" :

Les modèles de causalité les plus fréquents sont en fait grosso modo les mêmes que ceux utilisés par les "théories du Pacifique" exposés plus haut.

Robert E. Driscoll, dans "Economic issues in the Asian/Pacific Region" [in George C. Mitchell et alii (eds) "Asian-Pacific Dynamics - Economic, political, security -", World Affairs 
Council of Pittsburgh, 1985, pp. 18-25.], énumère les facteurs suivants à la base des performances économiques locales :

"1- the close cooperation between the private and public sectors; 2- stable economic policies; 3- growth in international trade; 4lack of external debt problems; 5- a balance between agricultural and industrial growth".

Mais cela n'a qu'un intérêt heuristique limité dans la mesure où l'agencement et la hiérarchie de ces facteurs ne sont quasiment jamais donnés.

\subsection{Détermination "mixte" :}

Des modèles plus sophistiqués et plus précis ont bien sûr été développés.

En général, ces types d'explications voient à l'origine de cette croissance une détermination mixte.

Ils constatent que cette dynamique de croissance est très dépendante de la conjoncture internationale en général (système économique mondial libéral avec démantèlement douanier et croissance du commerce international,...) et en particulier des Etats-Unis (accès au plus grand marché intérieur) ainsi que du Japon (investissements directs combinés aux transferts technologiques).

Ils ajoutent que cette dynamique est basée sur des conditions intérieures particulières :

combinaison d'un système politiquement autoritaire, d'une militarisation de la société, d'une forte pression sociale et de valeurs culturelles spécifiques dont les résultantes sont un taux d'épargne élevé, une allocation des ressources à 
l'investissement plus qu'à la consommation et une quantité de travail très importante.

\subsection{Approche culturelle :}

Pour arriver à dégager les éventuelles spécificités et "vertus" d'un modèle des quatre dragons, il est intéressant de confronter leurs réalités à la typologie des modèles de développement et de croissance.

\subsubsection{L'héritage du vieux Confucius.}

Dans un livre fort stimulant qui développe une partie des explications précitées ["La puce et le riz - croissance dans le Sud-Est asiatique", Armand Colin, Paris, 1985, 208 p.], Jean-Raphaël Chaponnière se penche sur la question.

- Il constate d'abord que "la croissance n'est pas arrivée là où l'attendaient les premiers économistes du développement" (...). En Asie, on ne se faisait guère d'illusions sur les "Etats mous" (Thailande, Malaisie, Indonésie, Philippines) ou sur les "Etats fantoches" qui, dans les années '50, survivaient des prébendes de l'impérialisme américain" (p. 175).

- Il passe ensuite en revue les différentes politiques de développement préconisées et utilisées :

stratégie d'import substitution avec protection temporaire des infant industries et accumulation du capital local;

stratégie du développement auto-centré pour briser le développement inégal inscrit dans le système des prix mondiaux et du marché mondial qui prolongent la domination coloniale; 
stratégie de l'internationalisation du capital et d'accueil des firmes multinationales dites "foot loose" dans un premier temps et que l'on parvient à stabiliser puis remplacer;

stratégie libérale où l'on choisit l'ouverture et la valorisation de ses avantages, comparatifs avec dévaluation, réduction des tarifs douaniers, promotion des exportations, plus le maintien de la concurrence à l'intérieur qui stimule les industriels et permet la remontée des filières.

- Il observe que les exemples ont précédé les modèles et que plusieurs de ces constructions théoriques sont des épures de l'expérience des pays de la Bande des Quatre. Il y distingue deux types de développement :

celui des villes-Etats (Hong-Kong, Singapour) ce qu'il appelle "le succès des entrepôts", centres commerciaux puis financiers, filiales ateliers qui réussissent leur révolution industrielle à la poursuite de créneaux high tech;

celui de Taiwan et de la Corée du Sud avec "la volonté de relever le défi japonais en suivant presque pas à pas le même cheminement".

Ces pays ont joué une politique de promotion des exportations tout en protégeant les secteurs qui sont devenus porteurs (industrie lourde et légère) et en encourageant la substitution aux importations. Ils ont choisi les exportations de produits manufacturés ce qui a permis d'éviter les grandes distortions de prix, l'emploi de technologies labour intensive et donc la création d'emplois. (Les investisseurs étrangers n'ont pas façonné la "vocation exportatrice" de l'industrie, mais la priorité des gouvernements a coïncidé avec la préoccupation de délocalisation des grandes firmes).[p. 154]. 
Les Quatre Dragons ont aussi profité d'une période d'expansion des échanges mondiaux sans précédent dans l'histoire. Malgré ce contexte et leur faible nombre, il est à noter que leur apparition a déjà provoqué la multiplication des mesures protectionnistes.

Pour J-R. Chaponnière, les explications économistes du développement sont décevantes.

- Il esquisse une approche culturelle du phénomène et énumère différents points communs que l'on peut tenir pour des conditions primordiales dans le "take off des Quatre Dragons" :

l'existence d'un pouvoir fort pour qui capitalisme n'est pas synonyme de laisser-faire et qui a su mettre en oeuvre la mobilisation de tous les facteurs de développement ( avec des coûts humains très lourds - cf. les "générations perdues" pour réaliser leur révolution industrielle en trente ans, ...);

$l^{\prime}$ approche pragmatique et non idéologique des problèmes économiques, et donc une grande flexibilité/adaptabilité;

l'attachement du pouvoir politique à l'essor économique, caractéristique sans doute de gouvernements plus technocratiques que politiques;

l'héritage confucéen considérant que le "gouvernement a reçu traditionnellement un mandat du Ciel pour exercer son pouvoir et assurer le bien-être de son peuple", où la recherche du consensus prime sur l'affrontement, où l'utilitarisme et le rationalisme ont une grande place (réduisant d'autant le choc mental dont est porteur la modernisation), où le respect de l'autorité prédispose à l'organisation du travail et à la productivité.

Deux précisions sont encore données pour apprécier cet 
apport du Confucianisme:

la religion ou la philosophie n'est pas le moteur des activités humaines, mais elle représente la cristallisation des valeurs sociales transmises; il n'y a pas de déterminisme religieux.

Selon notre auteur, le phénomène des Quatre Dragons est un événement historiquement marqué et non reproductible sur une large échelle. Il ne s'agit pas d'un modèle généralisable à l'ensemble du Tiers-Monde [p. 180].

3.4.2.La cohésion perdue et retrouvée du monde sinisé.

Ce modèle d'explication est partiellement repris dans l'ouvrage très fouillé $d^{\prime}$ un représentant de l'école française des relations internationales", Léon Vandermeersch, "Le nouveau monde sinisé" (PUF, Paris, 1986).

Comme le titre l'indique, le cadre de réflexion s'étend ici aux "Quatre petits Dragons" (Corée du Sud, Hong-Kong, Singapour, Taiwan), plus la Corée du Nord, la Chine et le Japon.

L'auteur voit à la réussite des quatre Dragons plusieurs facteurs favorables déjà mentionnés ça et là, c'est-à-dire, le fait $d^{\prime}$ être des économies à coefficient d'inertie assez faible vu leur taille; le soutien apporté par d'importants capitaux extérieurs; le fait d'être un "milieu sinisé"; l'avantage (quand on sait s'en servir) du développement tardif qui permet l'incorporation d'emblée des technologies les plus performantes tout en bénéficiant de l'avantage comparatif des coûts du travail et en économisant les coûts de développement [p. 23].

Mais le double objet de sa démonstration n'est pas là : 
- Le premier point serait l'existence "dans les croissances des pays sinisés, si diversifiées qu'elles apparaissent du fait des conditions particulières qui les ont affectées, $d$ 'un même dynamisme" et d'une même structure de développement! [p.37]

(Cette idée est parfois reprise ailleurs mais de façon élusive, lorsqu' on insiste sur le dynamisme "confucéen", sur le rôle très important pris par la diaspora chinoise dans les pays de l'ASEAN moins Brunei).

Il existerait des traits de similarité suffisamment accusés sur des composantes essentielles, à savoir, "une action vigoureuse de l'Etat, le rôle fondamental de l'agriculture, le privilège accordé à l'industrie lourde";

une structuration assurant la convergence des finalités du pouvoir étatique et du pouvoir économique, avec réglementation économique à finalité productiviste; un directionnisme consensuel puissamment inducteur" [p. 39].

Parallèlement, nous assisterions d'une part à la réhabilitation de la culture asiatique par les occidentaux (modèle dont il faut tirer des enseignements) et par les asiatiques eux-mêmes.

Le Confucianisme proprement dit avec sa trilogie famille, rite et mandarinat a disparu, mais a laissé un héritage profond dans les sociétés qui furent son berceau, c'est-à-dire le monde sinisé.

Ces sociétés en ont gardé des valeurs spécifiques : haute valeur conférée à l'éducation (niveau de scolarisation très élevé même si le niveau de développement économique est faible); tradition de frugalité, sobriété et prévoyance qui se traduit par des taux d'épargne record.

Ces sociétés sont marquées : 
par le communautarisme (profond anti-individualisme où l'homme n'existe qu'en tant qu'être social) qui s'est recentré de la famille vers l'entreprise [ p. 169];

par le ritualisme (qui se traduit par la prééminence d'une rationalité morpho-logique, par opposition à la rationalité occidentale de type téléo-logique, dont le but est la bonne harmonie de la société [ $p$. 176];

par le fonctionnalisme (où le contrôle de la société par l'Etat est dans l'ordre de la nature; où domine une tradition de gouvernement administratif et non politique [p. 86]; où l'action engagée par le parti dominant est en fait fort peu politique, visant principalement le développement de l'économie et de la modernisation [ $p$. 196].

Cet ensemble constitute un background extrèmement propice au développement économique.

- Le second point serait la restauration progressive de la cohésion du monde sinisé.

Le monde sinisé était jusqu'à la moitié du XIXe siècle au moins aussi cohérent que le monde occidental.

Il fut disloqué suite à plusieurs facteurs [p. 204] dont notamment l'action délétère directe de l'impérialisme occidental, puis du néo-colonialisme ainsi que les conséquences indirectes de l'importation d'idéologies occidentales antagonistes (nouveaux Etats fondés sur des régimes politiques idéologiquement ennemis).

Les techniques d'impérialisme les plus contraignantes se sont à la longue révélées trop coûteuses pour les Etats qui les avaient pratiquées, ce qui a débouché sur un désengagement assez large.

La dimension idéologique conflictuelle s'est également 
estompée et les véritables antagonismes inter-sinisés proviennent aujourd'hui principalement des séquelles de l'impérialisme japonais et des guerres civiles.

Enfin, la grande inégalité de développement économique pourrait progressivement se résorber avec le réveil du grand dragon, la Chine.

Bref, les facteurs de désintégration ont tous perdu de leur force, tandis que l'homogénéité culturelle de ce groupe est remise en exergue (du point de vue de la langue, de la forme de la société et de l'appareil conceptuel) et que se développe de nouvelles synergies de développement économique (complémentarité commerciale et complémentarité des investissements).

Pour L. Vandermeersch ce qui est en marche dans cette zone, c'est donc la restauration de la cohésion économique et politique du monde sinisé [p. 209] :

"Il existe dans le milieu des pays sinisés une spirale de développement plus puissante qu'ailleurs, à la pointe de laquelle se trouve le Japon, ..., qui tire derrière lui les autres pays sinisés, y compris l'énorme masse de la Chine populaire".

Il s'agit en fait de l'apparition d'une forme de civilisation égale à la civilisation occidentale pour les performances économiques, scientifiques et techniques, mais appuyées sur un système de valeurs, une conception de la société et une vision du monde différents.

On retrouve ici la problématique du déplacement des centres de gravité du monde mais selon un autre clivage. Ce clivage Monde sinisé/Monde occidental serait amené à peser de plus en plus sur les problèmes d'influences politiques, de règlements des contentieux économiques. 
Cela ramènerait l'axe des rapports Est-Ouest à sa simple dimension intra-occidentale!

Il n'est pas question de discuter ici d'une telle démonstration. Retenons simplement qu'elle postule, elle aussi, un modèle de croissance particulier, qui n'est transposable que de façon culturellement et donc géographiquement limitée, mais qu'elle est très optimiste quant à sa pérennité.

\subsection{Spécificité, transposabilité et pérennité, les trois grâces du Pacifique?}

Ces trois grâces combleront-elles demain l'ensemble du Pacifique de leurs bienfaits? En conclusion, il nous semble que l'on peut se prononcer de la façon suivante sur le triptyque spécificité / transposabilité / perénnité.

Les quatre "success stories" d'Asie Pacifique qui ont frappés si fort les imaginations sont bien spécifiques. Comme le dit M. Schmiegelow, ce modèle de croissance consiste en des "incomparable compositions of comparable components" (v. sa contribution dans le présent volume "The Asian Newly Industrialized Economies : a Universal Model of Action").

Elles ne semblent pas reproductibles ailleurs avec les mêmes ampleur et vitesse prises par ce développement économique, tout au moins pour le moyen terme. Leur généralisation à l'échelle du Pacifique est quant complètement inconcevable, même à très long terme.

Enfin, si la pérennité de ce rattrapage à une échelle relativement limitée est très plausible, elle n'est cependant pas garantie. 
Si l'on s'en tient au type d'explication "mixte", exposé plus haut, plusieurs événements récents tendent à montrer que certains de ces éléments peuvent disparaître ou s'affaiblir.

- Il y a plusieurs points d'interrogation qui pèsent sur les facteurs favorables exogènes.

Nous avons déjà indirectement parlé du point d'interrogation de la conjoncture internationale. Les progressions des exportations asiatiques proviennent en partie du déficit budgétaire et du déficit de la balance commerciale des USA. Ces déficits ne sont pas sans conséquences ( $\mathrm{cf}$. tensions économiques; montée des tentations protectionnistes américaines; nippophobie; résurgences nationalistes). Ils ne sont surtout pas éternellement soutenables et les solutions indolores sont "rares".

A titre d'exemple parmi bien d'autres, on peut encore mentionner le point d'interrogation des effets sociétaux et internationaux souvent imprévus des innovations technologiques.

Ainsi, les développements de l'automation dans la production, combinés à ceux des technologies de l'information dans les systèmes de gestion provoquent le retour de certaines filières industrielles vers les pays industrialisés occidentaux (cette automatisation permet une augmentation substantielle de productivité qui annule l'avantage comparatif de la rétribution du travail; le système de "stock zéro" qui diminue substantiellement les frais, exige une proximité géographique des fournisseurs; etc.).

- Il y a aussi des points d'interrogation qui pèsent sur les facteurs favorables endogènes.

L'hypothèse du dépassement contenue dans le scénario 
$\mathrm{du}$ "siècle du Pacifique" postule, parfois implicitement, une stagnation continue des autres entités (principalement l'Europe) mais surtout la possibilité de maintenir les spécificités de l'organisation sociétale à l'origine du take off quelque soit le stade de développement économique atteint.

- Or, les défenseurs de la "backwardness theory" soutiennent que parmi les avantages comparatifs des pays en retard de développement se trouvent précisement leur différence de développement. Lorsqu'ils ont la capacité et la possibilité de mobiliser capital humain et matériel et bénéficient de conditions extérieures favorables, ces pays peuvent croître beaucoup plus vite que le pays de tête car ils peuvent sauter certaines étapes et économiser les divers coûts de R\&D. $\mathrm{Au}$ fur et à mesure du processus de rattrappage, cet avantage s'amenuise fatalement et le tempo du processus s'en trouve considérablement ralenti. Les derniers pas seraient en fait les plus durs à franchir (ex. Le Japon, la Corée du Sud, Taiwan se sont rapprochés des USA mais sont encore à un niveau de productivité inférieur).

L'expérience du Japon tend à montrer qu'une super croissance ne peut se maintenir indéfiniment (durant la dernière décennie, la croissance de sa productivité n'a pas dépassé celles des meilleures performances européennes). Certains signes de ralentissement en Corée, à Taiwan (certes relatifs et sûrement conjoncturels) se font sentir [v. par ex. Alain Vernay, "Corée : les spasmes d'un dragon" Analyses de la SEDEIS, Paris, Sept. 1989].

- Par ailleurs, la démocratisation des régimes s'accompagne généralement d'une répartition ou d'une 
redistribution plus égalitaire des richesses. Avec la hausse du pouvoir d'achat vient une augmentation de la consommation mais aussi un développement des loisirs. Il s'agit d'un constat quasi historique : à mesure que les revenus réels augmentent, la demande, latente ou non, de temps libre augmente dans la population ... Cette évolution risque d'être d'autant plus rapide que ces pays sont aujourd'hui amenés à réorienter leurs politiques économiques vers une dynamique de croissance plus centrée sur la demande intérieure.

Une telle évolution est difficilement compatible avec une militarisation de la société et le maintien d'une logique strictement productiviste. Les changements qui devraient en découler meneraient à terme les dragons vers une évolution plus occidentale ou "post-industrielle" avec les conséquences que cela suppose en termes de type de croissance.

Bref, il est difficilement concevable que ces pays échappent aux problèmes structurels des sociétés "avancées" d'aujourd'hui (pyramide des âges, protection sociale, demande de loisirs, etc.) et que leur rythme de croissance n'en soit pas affecté.

4.La "Communauté du Pacifique" : avis de recherche en cours!

Cette "Communauté" semble être une sorte de monstre du Lochness ou de Yeti des relations internationales : tout le monde en parle sans l'avoir jamais vu; les indices tenus pour preuve de son existence peuvent être des indices de plusieurs choses. 
L'idée d'une Communauté Pacifique n'est pas neuve.

Elle avait déjà été lancée bien avant la seconde guerre mondiale. Sorte de doctrine Monroe du Pacifique, elle était surtout un paravent pour les ambitions impériales nord-américaines et japonaises dans la région, qui prirent des formes surtout commerciales et économiques dans le premier cas, qui finirent dans les aventures militaires et les annexions sanglantes de la "sphère de co-prospérité asiatique" dans le second cas.

Le Pacifique de l'après-guerre est déchirée par la guerre froide ou mis sous tutelle des puissances victorieuses.

Dans le même temps, un des autres faits marquants de la période sont les premiers succès de l'intégration européenne. L'Europe est une mosaïque de peuples aux langues, aux valeurs et aux cultures différentes, ayant un long passé commun de conflits sanglants.

Un fort mouvement favorable à l'intégration de ces entités se développe pour différentes raisons après la seconde guerre mondiale. Les formules envisagées échouent les unes après les autres jusqu'à l'adoption d'une démarche néo-fonctionnaliste (c'est-à-dire combinant tactique fonctionnaliste et finalité fédérale) qui débouche sur la création de la "Communauté Economique Européenne". Celle-ci n'est pas une fin en elle-même. Elle est la première étape d'un dynamique menant au "spill-over" vers l'intégration générale. A travers cette nouvelle institution, les Etats signataires établissent "les fondements d'une Union sans cesse plus étroite entre les peuples européens" (préambule du traité de Rome CEE).

Le terme "Communauté" n'est d'ailleurs pas chosi par hasard. Il sous-entend en effet l'existence d'une dimension psychologique "affective" dépassant les simples intérêts économiques réciproques, un niveau de cohésion supérieur, un sentiment d'appartenance à une même entité sociale. Dans le 
domaine des sciences politiques, ce terme fut donc utilisé de façon inédite pour désigner un stade nouveau de l'intégration et le schéma organisationnel particulier qui s'y attache (un nouveau type d'association d'Etats - empruntant des éléments aux systèmes de la Confédération, de la Fédération et même de $l^{\prime}$ Etat unitaire - que F. Dehousse qualifiait de "découverte scientifique majeure des sciences politiques" [in "L'Europe et le Monde", Librairie Générale de Droit et de Jurisprudence, Paris, 1960, p. 375].

Cette formule fonctionne bien et se révèle être rapidement très profitable pour ses membres. La Communauté européenne devient tout naturellement un modèle d'intégration à suivre.

\subsection{Toile de fond Pacifique.}

L'idée d'une Communauté Pacifique renaît alors, notamment au Japon.

Mais le Pacifique n'est pas l'Europe. Les entités concernées en Europe ont des systèmes politiques semblables et un niveau de développement économique raisonnablement homogène; les différences ethniques et religieuses y sont plus faibles et posent moins problème; le nationalisme s'y est émoussé et est partiellement discrédité; elles appartiennent toutes à la même civilisation au sens large; elles bénéficient d'une continuité géographique sur un espace total réduit.

Il est toujours possible d'établir une "Communauté" si une volonté politique suffisante existe, mais cela n'est jamais facile. Et dans le cas du Pacifique, les obstacles étaient et sont encore énormes!

Il s'agit d'un ensemble extrêmement hétérogène et parfois conflictuel tant au niveau militaire, idéologique, politique, économique, technologique, social, culturel, historique, religieux, ethnique,... Plusieurs civilisations se sont développées dans le Pacifique. S'il est vrai qu'une 
acculturation est en cours, elles n'en ont pas pour autant disparu. Non seulement elles s'y juxtaposent encore en grande partie, mais le nationalisme y est resté vivace ou renaît.

L'utilisation du terme "Communauté" par les partisans d'une intégration du Pacifique reste donc, hier comme aujourd'hui, "techniquement" un abus de language ou un voeu pieux, dans la mesure où les éléments de la définition énoncée ci-dessus ne sont pas réunis en l'espèce.

\subsection{Projets institutionnels.}

Le constat qui précède est confirmé par l'examen des actions concrêtes qui pourraient attester de l'existence tangible d'une Communauté Pacifique.

Globalement, il faut reconnaître que le problème de son établissement a été très largement et très souvent discuté depuis au moins 25 ans, et que l'on s'y intéresse aujourd'hui au Japon, en Extrême-Orient, en Amérique du Nord, en Amérique centrale, en Amérique latine, en Australie,...

Dans ces discussions, les dimensions politiques et militaires furent écartées d'emblée des projets d'intégration pour des raisons évidentes.

Un nombre assez important de projets présentés comme un premier pas dans l'établissement d'une Communauté du Pacifique se sont dès lors succédés au niveau de l'économique. Les Japonais, par des moyens plus ou moins indirects ont souvent été parmi les initiateurs de ces projets. Les milieux d'affaires et les milieux académiques japonais ont été les premiers à étudier sérieusement le problème. Le professeur Kiyoshi Kojima de l'Université d'Hitotsubashi proposa en 1965 la création d'une "Pacific Free Trade Area" ("PAFTA" sur le modèle de l'EFTA") entre l'Australie, la Nouvelle-Zélande, les 
USA, le Canada et le Japon. Mais cela signifiait un accroissement des déséquilibres des balances de paiement et aucun accord ne fut possible.

4.2.1.Les premières réalisations : PTDC et $\mathrm{PBEC}$ en 1968.

Il faut attendre 1968 pour assister aux premières et timides réalisations en la matière.

- D'une part, nous avons l'organisation par le "Japan Economic Research Center" de la première "Pacific Trade and Development Conference" (PTDC) réunissant des universitaires et des spécialistes. Avec le soutien du Ministre japonais des Affaires étrangères, Takeo Miki, ces réunions devinrent annuelles et rassemblent des académiques de nombreux pays de la région, les Américains, les Australiens et les Japonais se montrant apparemment plus actifs. (Aux USA par exemple, multiplication des instituts tels East West Center, Pacific and Asian Affairs Council (Hawai); the Pacific forum; the Mansfield Center for Pacific Affairs (Montana); the Pacific Basin Institute (Santa Barbara - California); ...).

Les discussion du PTDC se concentrent sur la faisabilité des différentes solutions, de leurs avantages et inconvénients respectifs. L'idée d'une Organization for Pacific Trade and Development (OPTAD) fut longtemps à l'ordre du jour et fut notamment l'objet d'un rapport des professeurs P. Drysdale (Australie) et H. Patrick (USA) décrivant ses raisons d'être et ses objectifs :

"First, it would provide a more effective safety-valve,given the high existing levels of interdependence for the discussion of trade and economic grievances among Pacific countries in a national and cooperative atmosphere. Second, it would 
provide a stimulus to investment and aid flows for the developing countries of the Asian-Pacific region and a framework for improvement in the structure and quality of their aid, investment and trade relations with the developed countries in the Pacific. Third, it would provide a forum for consultation and discussion about the longer term developments and economic transformation of the region. And finally, it would offer a more secure framework of economic alliance among the countries of Asia and the Pacific".

Jusqu'ici ce projet n'a pas encore été repris et soutenu par un seul gouvernement de la région!

- D'autre part, nous avons la création par et pour les milieux d'affaires du "Pacific Basin Economic Council" (PBEC) où sont représentées à ce jour des organisations provenant de 16 pays de la région. Ce Conseil, dont la périodicité des réunions est variable, se veut le porteparole des désirs et des interrogations du monde économique vis-à-vis du bassin Pacifique.

Stuart Kirby [op. cit., p. 23] le décrit comme "the clearest advocacy of the Pacific Basin movement (...). This is an open organisation, characteristically of business firms and leaders, chambers of commerce and ind ustry and the like. It proceeds in what may be described as a somewhat Rotarian atmosphere of togetherness and goodwill, contact, exchange of ideas and collaboration for mutual benefit, ...".

4.2.2.Une troisième organisation : le PECC.

Plus de 10 ans après, une troisième organisation vient se rajouter aux "PTDC" et "PBEC". 
Le premier Ministre japonais, Masayoshi Ohira, au cours de sa campagne électorale, avait mentionné son intention d'étudier les possibilités de développement de la coopération entre les pays du Pacifique. En 1979, il mit sur pied un groupe d'étude privé sur la question. Ses conclusions étaient que la formation d'une Communauté était à la fois désirable et possible, s'il était tenu compte du dynamisme de la région et de sa diversité; que cette communauté devait être un modèle sui generis et qu'elle devait, particulièrement dans les relations Nord-Sud, servir les intérêts globaux aussi bien que régionaux; qu'elle devait, non remplacer la cooperation bilatérale ou multilatérale existante, mais coexister avec elle [v. Mitsuro Donowaki, "The Pacific Basin Community - A Japanese overview", Asia Pacific Community, Tokyo, Winter 1982, pp. 15-29].

Soutenu par le premier Ministre Australien, Malcolm Fraser, cette initiative déboucha en 1980 sur la tenue d'un séminaire à l'Australian National University rassemblant académiques, hommes d'affaires et des officiels présents à titre privé, qui décida la création du "Pacific Economic Cooperation Conference" (PECC) qui est chargée d'organiser des réunions approximativement annuelles et d'établir des task-forces pour l'étude de différents sujets.

$\mathrm{Ce}$ nouveau forum qui reste donc non-gouvernemental, est censé jouer plus ou moins le rôle qui aurait été dévolu à une "OPTAD". Selon Kumao Kaneko, directeur du Japan National Committee for Pacific Economic Cooperation, "the PECC has developed into a forum for the free and informal exchange of view in a clublike atmosphere" " "A new Pacific initiative : strengthening the PECC process", Japan view of international affairs, Vol. 2, No 1, summer 1988, p. 76].

L'hypothèse de travail du PECC concernant les frontières de l'entité Pacifique est longtemps restée le "5+6+X" (rappel : 6 
pays développés USA, Canada, Japon, Australie, Nouvelle Zélande +5 ASEAN + X Nics).

A ce jour, la Chine est devenue membre du PECC, tandis que l'URSS et différents pays d'Amérique du Sud assistent à ces réunions seulement à titre " $\mathrm{d}$ 'invités ou d'observateurs" (malgré la demande de certains - Mexique, Pérou, Chili -de devenir membres à part entière).

A l'issue de la réunion de Vancouver en 1986, une déclaration clarifiant les buts et activités du PECC a été adoptée :

"Participants in the Pacific Economic Cooperation Conference (PECC) from business and industry, government, academic and other intellectual circles, believe that realization of the full potential of the Pacific Basin depends enhanced economic cooperation based on free and open economic exchanges and in a spirit of partnership, fairness and mutual respect.

"We are mindful of the shared interest in promoting economic cooperation among the economies in the region to bring about greater economic and social benefits and well-being for our respective peoples and contribute to the stability, prosperity and progress of the entire region.

"Participation in the PECC process will depend upon a commitment to economic cooperation in the Pacific. PECC participants, who have extensive economic activities in the Pacific, will seek to achieve increased regional economic cooperation and interaction, while recognizing both the realities of and the benefits accruing from global inter-dependence and continuing to encourage economic cooperation and interaction with other nations and regions".

Mais qu'y-a-t-il derrière les grandes déclarations d'intentions?

L'évaluation lapidaire qui suit nous semble correcte [Michael 
b. Yahuda, "The 'Pacific Community' : not yet", The Pacific Review, Oxford University Press, Vol. 1, N 2, 1988, p. 119] :

"Even the PECC's participants who are grouped into national delegations take part only in a semi-official basis. Such declarations or resolutions that it may pass are not binding on the governments concerned eventhough these are reached by consensus implying that each delegation may exercise a veto. So far the PECC has confined itself to issuing taskforces and establishing study groups"

En dehors des trois organisations qui viennent d'être mentionnées, il n'existe pas de structures trans-Pacifique (quelle que soit la définition de cette entité).

\subsection{Le bilan en 1990 .}

Quel constat peut-on dès lors faire concernant la question de l'existence d'une Communauté du Pacifique?

L'établissement d'une coopération inter-Pacifique pouvait sembler dans le passé sinon sans objet, du moins sans intérêt immédiat.

Depuis 25 ans, suite au dynamisme exceptionnel et à l'ouverture sur l'extérieur de plusieurs entités de la région, un nombre grandissant de voix soutient qu'une coopération serait, dans son principe même, grandement et mutuellement bénéfique.

Plusieurs pays qui étaient restés à l'écart de ce mouvement s'intéressent aujourd'hui à la question.

Mikhail Gorbatchev dans un discours retentissant à Vladivostok le 28 juillet 1986 a déclaré que l'URSS se considérait comme un Etat du Pacifique, a présenté un 
programme d'action vis-à-vis de cette région et s'est déclaré prêt à s'associer aux réflexions concernant les fondements d'une coopération économique dans le basin Pacifique. Dans l'article mentionné plus haut, Bogomolov s'insurgait de la stratégie globale de l'impérialisme (capitaliste) visant à isoler politiquement et économiquement les pays socialistes de cette évolution et de les empêcher de participer à la division du travail à l'échelle régionale. Il ajoutait que "le bassin Pacifique joue un rôle de plus en plus grand dans les plans d'édification des deux grandes puissances socialistes asiatiques (!) : l'Union soviétique et la République populaire de Chine".

Dans le même temps, diverses associations de pays d'Amérique latine se sont également formées dans l'espoir de devenir partie prenante dans les "institutions Pacifiques" existantes [v. Francisco Ornego Vicune "Pacific Co-operation : the view from Latin America; The Pacific Review, Oxford University Press, VoL. 2, N 1, 1989, pp. 57-67 ].

De nombreux efforts et initiatives ont été déploiés en ce sens de la part de membres de diverses "élites" (businessmen, académiques, hommes politiques) provenant principalement des Etats régionaux membres de l'OCDE. Les personnalités favorables à "une" coopération ne manquent donc pas.

Dans quels domaines précis et selon quelles modalités, là le problème reste entier.

Il n'est en tout cas pas question d'un transfert de compétence, l'inter-gouvernemental pur seulement est à l'ordre du jour. Quant au passage à l'acte ...

Comme nous l'écrivions en novembre 1989 dans une note pour la Commission des Communautés européennes "Japanese policy toward regional integration", if we consider the past record of Pacific integration on the institutional level, there is a sharp contrast with the ambitious aims we were entertained 
with. The ongoing process remains more or less at the level of informal talks and pressure groups activities.

Si plusieurs gouvernements se disent intéressés par l'idée et financent plus ou moins indirectement des discussions ou recherches sur le sujet, aucun en 25 ans n'a émis formellement l'opinion que le temps était venu de convoquer une conférence intergouvernementale pour discuter du sujet. La proposition d'une simple réunion ministérielle des pays "membres" du PECC émise par la Corée du Sud resta, par exemple, sans écho.

\subsection{L'impasse et ses raisons.}

Les raisons premières de cette situation sont non dites mais semblent assez claires.

D'une part l'aspiration à une Communauté du Pacifique reste une aspiration technocratique et "affairiste". On ne voit pas comment cela pourrait changer du fait de l'extrême hétérogénéité (à la fois objective et vécue comme telle) de cette zone géographique. Cette aspiration est elle-même très inégalement répartie.

D'autre part, la mise en oeuvre d'une intégration régionale exige qu'un nombre minimum de conditions (notamment matérielles) soient réunies et elles ne le sont pas.

4.4.1.Petite analyse comparative.

Pour expliciter cela, on peut procéder par comparaison avec la construction européenne qui reste le seul exemple contemporain de large réussite en ce domaine.

- P.Soldatos a élaboré une vision synthétique et précise des origines des Communautés européennes ["L'explication 
historico-politique de la génèse des C.E.", in Lasok Y. et Soldatos P. (ed.), "Les Communautés européennes en fonctionnement", Bruylant, Bruxelles, 1981, p. 41 et s.]. Pour lui, les déterminants fondamentaux de ces origines sont au nombre de quatre:

"1. La crise économique et les besoins $d u$ mode de production des unités politiques concernées;

2. la crise socio-politique;

3. la crise diplomatico-stratégique et militaire;

4. les images créatrices des élites dirigeantes ayant conduit à l'élaboration d'un projet intégratif aux traits thérapeutiques et aux potentialités dynamiques de croissance systémique".

ㅁ Il y ajoute sept conditions favorables au déclenchement du processus :

"1. la symétrie dans la taille économique des partenaires;

2. la compatibilité des valeurs (socio-culturelles et économico-politiques);

3. l'existence d'un réseau de communications sociales;

4. les influences externes;

5. L'existence d'un pluralisme socio-politique au sein des partenaires;

6. l'existence de groupes pro-intégratifs organisés;

7. la capacité d'adaptation et de réponse des gouvernants".

4.4.2. Faiblesse des déterminants fondamentaux.

En ce qui concerne les déterminants fondamentaux, il n'y a que le point 1. et partiellement le point 4 . dont peut bénéficier l'intégration du Pacifique. 
En reformulant cette réponse, on peut envisager le problème de l'intégration Pacifique sous l'angle de ses déterminants potentiels internes et externes.

- Les déterminants internes seraient :

- une solution et une assurance aux crises politico-militaires plus ou moins en sommeil aujourd'hui.

Apaisement des vieilles haines historiques (surtout entre le Japon et ses anciennes colonies ainsi que les ou pays occupés -Corée, Philippines, Chine, ...); système de sécurité collective, garantisant le statu quo des situations nationales et régionales à l'image de ce qu'à presque complètement réussi à ce jour l'ASEAN à son niveau (càd fin des soutiens extérieurs aux mouvements séditieux et de l'octroi de sanctuaires pour guerrillas révolutionnaires, règlements de vieux conflits territoriaux,...).

- une optimalisation économique.

Allocation des ressources et division du travail plus performante au niveau régional;

exploitation des économies d'échelle;

développement d'une base de repli régionale en cas de repli de l'économie mondiale, de montée du protectionnisme, d'effondrement du système libéral, de constitution d'autres forteresses régionales,...

- une réponse vague et généraliste à l'overstrech de structures politiques nationales.

- Les déterminants externes seraient essentiellement d'ordre économique (dont les aspects internes-externes sont difficilement dissociables - v. supra).

On ne voit pas ici de menace extérieure perceptible 
(comme celle soviéto-marxiste que percevait l'Europe après la seconde guerre mondiale).

Des déterminants existent donc mais on n'observe pas en l'espèce l'intensité qui caractérisait les intégrations qui ont atteint le stade de la mise en oeuvre.

\subsubsection{Les conditions favorables manquantes.}

Pour ce qui est des conditions favorables, elles font quasi toutes cruellement défaut.

En ce qui concerne la symétrie, la compatibilité, le pluralisme, cela se passe de commentaires. Le réseau de communications sociales reste aujourd'hui très lacunaire.

Par contre nous l'avons vu, des groupes pro-intégratifs existent, sont organisés et disposent de moyens.

Reste en fait à examiner le point 4 de ces "conditions favorables" au déclenchement du processus d'intégration : les "influences externes", auxquelles nous pouvons ajouter les "fédérateurs internes".

Par "influences externes", P. Soldatos fait référence dans le cas européen à l'existence de fédérateurs externes (USA, G-B). On peut y ajouter la question de l'existence de fédérateurs internes, c'est-à-dire les "moteurs" de l'intégration prêts à faire les premiers pas, à supporter des surcoûts au départ,...

Qu'en est-il dans le Pacifique, au vu des positions officielles des Etats concernés? 
- L'ASEAN et les petits pays.

Les pays de l'ASEAN gardent une position en retrait par peur de voir leur association diluée, par peur de voir leur dépendance accrue vis-à-vis $d^{\prime} u n$ condominium américano-japonais.

L'ASEAN ne veut pas d'un grand protecteur.

Mais l'ASEAN, vieille zone "naturelle" de l'expansion commerciale du Japon, est aujourd'hui très dépendante du Japon pour ses importations et ses exportations. Les pays qui en sont membres souffrent tous de déséquilibres importants de leur balance d'opérations courantes avec le Japon.

Sa dépendance est encore accrue par le fait que l'ASEAN reste de loin le premier bénéficiaire de l'aide au développement du Japon, avec plus d'un tiers des montants de l'ODA (que le Japon compte porter à 10 milliards de \$ par an dans le cadre de son "International Cooperation Initiative"). Cette aide est de plus dénoncée comme étant très paternaliste et comme étant un outil commercial (business tool) en raison de sa très faible part de dons.

Pour ces raisons, l'association recherche un développement de ses relations avec d'autres entités et notamment avec la Communauté européenne pour rééquilibrer sa situation [v. Europe information n 92/88 "The European Community and ASEAN", avril 1988, Commission of the European Communities, pour un exposé succint de leurs relations commerciales, de leur coopération économique ou de développement].

Le tableau des investissements directs approuvés par les "National Investment Promotion bodies des pays de l'ASEAN, montre que l'Europe n'en est d'ailleurs pas absente : 
en Indonésie, les pourcentages des investissements cumulés de 1967 à 1987 sont respectivement de $12 \%$ pour la C.E., de $5 \%$ pour les USA, de $30 \%$ pour le Japon; aux Philippines, nous avons $17 \%$ pour la C.E., $15 \%$ pour le Japon, $36 \%$ pour les USA;

pour l'année 1986, en Thailande, nous avons $27 \%$ pour la CE + AELE, 3,5\% pour les USA, $57 \%$ pour le Japon;

à Singapour, pour l'année 1987 , nous avons $16 \%$ pour la CE, $41 \%$ pour le Japon, $37 \%$ pour les USA; etc.

Dans le même temps, les membres de l'ASEAN connaissent un dilemme grandissant du fait de leur peur que le rapprochement entre la Chine (qui est un concurrent direct dans beaucoup de domaines) et le Japon ainsi que les USA ne se fasse à leur détriment.

L'ASEAN désire donc aussi le maintien d'une coopération privilégiée avec le Japon, mais sous d'autres formes. Les membres de l'Association sont donc à la recherche de difficiles équilibres entre ces deux objectifs difficilement conciliables en pratique.

Les autres petits pays se méfient également d'un jeu dont les règles seraient dictées de facto par les grands.

Ils craignent de perdre leur marge de manoeuvre dans un cadre multilatéral obligé (considérant qu'un cadre bilatéral permet de jouer plus efficacement les grands les uns contre les autres). D'autres préfèrent enfin tenter la solution du free-rider sans obligations, du suiveur à moindre coût.

Pour de raisons très diverses donc, il n'y a pas de fédérateur, même potentiel, parmi les pays qui précedent. 
ㅁ Le Japon.

Les positions exprimées par Saburo Okita, Conseiller auprès du Ministre Japonais des Affaires étrangères, ancien ministre lui-même ["the outlook for Pacific cooperation and the role of Japan", Japan Review of International Affairs, spring-summer 1987, pp. 2-16] ou par Masao Fujioka, Président de la Banque Asiatique de Développement ["Toward Globalism" delivered at the symposium "Agenda for Asia, Agenda for the world", Osaka, Oct. 1988 ] sont assez représentatives du discours officiel japonais.

On y parle du "advent of the age of the Pacific". On se limite a des constats et projections "in terms of output and trade", de contribution de la région au progrès/développement de l'humanité mais sans conclusions triomphalistes d'ordre géopolitique concernant un leadership mondial, un modèle impérial à venir. Ils parlent de responsabilité et des devoirs qui incombent au Japon en tant que principale puissance économique régionale $d$ 'Asie-Pacifique dégageant d'importants surplus de balance des paiements.

"The challenge will be for Japan to take the leading role in trade and investment as well as in economic cooperation in Asia" [Masao Fujioka, P. 19 ].

On évoque "a Japanese Marshall plan", utilisant l'énorme épargne et surplus de comptes courants japonais pour satisfaire les besoins de capitaux des pays en développement de la région Asie-Pacifique.

"Our task is to manage and develop this (economic) interdependence (in the region) in such a way as to foster a 
better balance among countries and ensure the region' stability and prosperity. The most important aspects of this task are maintenance of an open economic system and industrial restructuring" [Okita S., p. 13 ].

Nous avons déjà esquissé plus haut les raisons de ce profil officiel relativement bas.

Les ambitions réelles du Japon apparaissent en filigrane des livres blancs publiés par le département d'Etat aux Affaires étrangères, le MITI, le JETRO, dans des publications académiques ou les conversations off record. Le Japon contemporain sait sa force et, du point de vue de la realpolitik, il est dans "l'ordre des choses" qu'une nation exploite les avantages de sa puissance.

En clair comme le dit Prasert, "Japan wants to be secund to none in Asia Pacific"... [op. cit. p. 573 ].

L'ambition de fédérateur interne, de locomotive de l'intégration ne lui manque pas. De nombreuses initiatives semi-officielles en émanent dont se dégage une vision plutôt volontariste du processus.

Le Japon est d'autant plus amené à développer un rôle actif au niveau international que la croissance de son PNB pour 1989 avoisine les $5 \%$ avec une inflation de $3,3 \%$, et que, combiné aux pharamineux excédents de sa balance commerciale et à son taux d'épargne moyen, cela lui pose des problèmes de recyclage de capitaux. (En janvier 1990, le Japon entrait dans son 38ème mois de croissance consécutif, s'approchant de la durée de son 2 ème boom économique le plus long - Iwata de juin 1958 à décembre 1961 ). Le Japon est devenu un gros exportateur de capitaux et est à la recherche de possibilités d'investissements à l'étranger qui peuvent être 
raisonnablement garantis.

De plus, les entreprises japonaises doivent aujourd'hui se mondialiser, se multinationaliser en créant de véritables filiales et unités de production locales, alors que jusqu'ici, outre les placements fonciers, les investissements japonais étaient destinés à assurer l'accès à des marchés (réseaux de distribution, usines tournevis, etc.) ou à maîtriser l'amont (matières premières et produits semi-finis provenant des pays de l'ASEAN et des NIES).

Il ne faut bien sûr pas voir derrière toute action japonaise une manifestation de volontarisme, d'une soif de puissance ou de son "traditionnel" et "irrésistible" expansionisme.

Nous l'avons déjà mentionné, le Japon est également l'objet de sollicitations dans la région et il n'y a pas que le désir de l'ASEAN d'une coopération privilégiée. Depuis les années '70, le Japon se voit régulièrement accusé de toutes parts de pratiquer une politique de "free-rider", $c^{\prime}$ est-à-dire de tirer les marrons du feu, de profiter des efforts des autres sans contreparties. Suite à ces pressions, le Japon a été amené récemment à développer son image politique, à se déclarer prêt à supporter sa part de "responsabilité internationale".

Cela ne va d'ailleurs pas sans lui poser des problèmes surtout dans le domaine militaire. L'insistance des USA sur le problème du "burden sharing" crée des tensions entre "defence build-up" et "self-defence". Le Japon a déjà fait différents gestes vis-à-vis des USA, refusant l'option "substituting" mais acceptant l'option "supplementing" notamment en augmentant son aide au développement en faveur des "pays de front" - Thailande,Pakistan, Turquie - [ v. Adelphi paper N 212, p. 44 ]. 
Last but not least, si le Japon possède bien la volonté et la stature d'une locomotive de l'intégration Pacifique, il ne peut assumer seul ce rôle.

Il y a encore ce que l'on pourrait appeler le syndrome du "vaincu/criminel de guerre". Les rancoeurs dans la région sont plus vivaces qu'il n' y peut paraître à première vue. Divers événements en attestent : manifestations anti-japonaises récurrentes lors de visites de Premiers Ministres japonais; polémiques à l'occasion de la mort de l'Empereur Hiro-Hito sur les responsabilités dans la guerre; violentes réactions suite aux modifications édulcorantes dans l'enseignement de l'histoire de la 2ème guerre mondiale dans les manuels japonais (accusations de mini-révisionnisme; "les vieux démons ne sont pas morts").

Cette méfiance est maintenue et actualisée dans les pays d'Asie Pacifique par l'identification qui existe entre les grandes entreprises "étrangères" exploiteuses et le Japon, vu le caractère dominant et massif des investissements de Zaibatsu (suspicion de "spiritual revival in military memory"; la vieille ambition renait, ses moyens ont changé).

La situation du Japon peut se résumer par l'extrait suivant tiré d'un précedent article : Japan's foreign policy typifies "the schizophrenic" situation facing potential initiators and leaders of a pacific community. Because of its double personality (member of the western world politically, economically and militarily; Asian nation geographically, historically, culturally, ...), Japan is singing on several registers at the same time.

We have the International Cooperation Initiative and its three pillars (cooperation for peace, expanding ODA, promotion of international cultural exchange),..., which 
could be summarized by the following sentences often repeated : "sharing global responsibility", "towards globalism".

But, on the line of the Fukuda doctrine, the Asia Pacific region is put on top of the Japanese agenda of priorities. Despite a formal modesty constantly repeated, a huge economic power like Japan has an understandable desire to secure its position and to regain its past prestige. There seems, so far, to be no consensus in Japan on the international leading role Japan should have to play.

But it will be foolish to consider this as a long term situation. And when this consensus will be reached, there surely will be much more convincing initiatives toward the Pacific basin and Asia Pacific issues.

En conclusion donc, le Japon a déjà activement et délibérément contribué à l'émergence d'un pôle Asie-Pacifique, d'une zone Yen, dont la présente organisation lui profite.

L'établissement d'un ordre plus large de même nature est assurément un autre de ses objectifs. Mais comme il ne peut être question ni de supplanter les USA ni de reprendre les funestes politiques du passé, c'est la solution d'un duopole paternaliste à l'échelle du Pacifique $(5+6+x)$ avec répartition des rôles entre le Japon et les USA qui serait la plus avantageuse pour lui et donc la plus tentante.

De là, la méfiance qui existe chez les partenaires et qui ne pourrait que se renforcer si le Japon se mettait à jouer un rôle plus pressant d'initiateur.

La République fédérale d'Allemagne, qui a bien des points communs avec le Japon, est devenue un des fédérateurs internes de l'intégration européenne parce qu'elle en avait la capacité, la volonté mais surtout parce qu'elle en a eu la 
possibilité grâce à la politique française, à la bénédiction des fédérateurs externes et aux formules institutionnelles qui ont créé différents contrepoids.

Le Japon vis-à-vis d'une Communauté du Pacifique possède sans doute la même capacité et volonté mais ne bénéficie pas des autres facteurs "habilitants".

Donc, le Japon ne peut être à l'échelle du Pacifique un fédérateur affiché (ni interne, ni externe).

\section{a Les USA}

Les positions officielles des Etats-Unis vis-à-vis d'une intégration pacifique, à la fois ambigues et fluctuantes, ne sont pas sans rappeler ses positions vis-à-vis del'intégration européenne.

Au commencement était une position sereine et bienveillante, sorte de "benin neglect" vis-à-vis d'un mouvement qui concerne sa péripérie. Les Etats-Unis sont "la" super- puissance, le centre du monde et ne peuvent qu'être indirectement et accessoirement impliqués dans des mouvements régionaux.

Leur stratégie est avant tout planétaire et globale. Les USA se voient comme les USA bien plus que comme un pays de telle ou telle région et ses dirigeants ne semblent pas intégrer leur pays dans le Pacifique sinon par une réthorique ambigue. On parle volontiers d'une interdépendance croissante des économies, on exige l'ouverture des autres marchés (la PAFTA redevient attractive), mais pour le reste une grande prudence est de mise.

Un exemple parmi beaucoup, extrait d'une communication de Gaston Sigur Jr., Assistant Secretary for East Asian and Pacific Affairs ["US and East 
Asia-Pacific relations : the challenges ahead", current policy N859, US Dept of State, Washington D.C., 1986 ] :

"... I detect a significant parallel between the pioneering spirit of our American West and that sense of entrepreneurship and dynamism which is rapidly transforming the East Asian and Pacific region into a leading center of global commerce".

Cette position bienveillante s'est ensuite transformée en sentiments mitigés ("mixed feelings") au fur et à mesure du "rattrappage" opérés par certains seconds rôles et avec l'ébranlement de la belle certitude d'être le maître du jeu. Tant que l'intégration du Pacifique pouvait signifier une nouvelle répartition des tâches laissant aux USA la haute main sur l'essentiel (un burden sharing sans power sharing), les USA étaient prêts à jouer le rôle du fédérateur externe à l'instar des conceptions d'un Churchill et de la Grande-Bretagne après 1945 vis-à-vis de la construction européenne.

L'admiration répétée devant les succès économiques du Japon, présenté comme un modele à suivre avec son harmonie sociale et son capitalisme confucéen, ..., a progressivement fait place à la peur du déclin inexorable, mais aussi à la conviction d'être le dindon de la farce. [v. Bernard K. Gordon, "Politics and protectionism in the Pacific", Adelphi papers n228, IISS, spring 1988, 82 p.; p. 44 "The American position"]; [v. Robert A. Scalapino, "Regionalism in the Pacific : prospects and problems for the Pacific basin", in the Atlantic Community Quarterly, summer 1988, Vol. 26, n 2, p. 174 et s.].

Lorsque Sony rachète Columbia, lorsque Mitsubishi Estate rachète le Rockfeller Center, (ce qui a une importance plus symbolique qu'économique), ce n'est 
pas le grand vent du Pacifique qui souffle mais une nippophobie grandissante et avec la sensation de se faire dévorer vivant par ces dragons asiatiques plus adversaires que partenaires.

Le Pacifique pourrait d'ailleurs à cet égard être le centre à venir des conflits commerciaux les plus empoisonnés plutôt que le centre du monde.

Les USA $n^{\prime}$ agissent pas comme un fédérateur interne et ne semblent pas être disposés à jouer un tel rôle.

Ils ne sont aujourd'hui qu'un fédérateur externe relatif.

口 L'Australie.

Pour mémoire, ce pays se montre très actif et intéressé par un tel processus (depuis le relâchement des liens du Commonwealth et l'entrée de la Grande Bretagne dans la Communauté européenne).

Sa taille lui permet de jouer un "supporting role", celui d'honnête courtier, de Monsieur bons offices, de médiateur, mais pas celui de moteur principal du mouvement.

En conclusion, les conditions de base nécessaires au "décollage" d'une organisation à l'échelle du Pacifique ne sont pas réunies et, semble-t-il, pour longtemps encore, sauf crise majeure comme une récession économique mondiale. 
Certains argumentent que l'intégration du Pacifique peut continuer à progresser sans expressions institutionnelles c'est-à-dire sans organisation structurée.

Par exemple, Michael B. Yahuda [op. cit., p. 126 ] écrit,

"there is a paradoxe that it is precisely when the dawn of the long heralded Pacific Century has arrived that the prospects for giving it organisational expression would appear to be receding rather than growing in strength. The growth of interdependency and the continued economic vitality and technological dynamism inherent in the Pacific basin has continued in the absence of economic community structures. Domestic factors underlying the economic development of the Pacific rim countries ... combined with the specific characteristics of interdependence have not required a formal regional organisational expression in order to have accomplished the remarkable economic achievments made so far".

Cette vision des choses ne manque pas de pertinence. Nous y voyons cependant une objection fondamentale:

est-il correct de parler d'une intégration régionale spécifique (c'est-à-dire allant au-delà du phénomène d'une croissante interdépendance générale et à l'échelle mondiale) dès lors que non seulement le terme "région" n'est qu'une abstraction géographique, mais surtout qu'il n'existe aucun engagement réciproque spécifique, aucune structuration spécifique des relations dans cette entité ?

\section{Les autres réalités régionales.}

Nous soutenons que la réalité de cette région Pacifique est contestable à bien des égards.

Que cette Communauté du Pacifique, en caricaturant, n'est encore d'un point de vue concret qu'une sorte de Chambre de 
commerce.

Que les phénomènes réellement nouveaux apparaissent lorsqu'on désagrège plus avant cette zone géographique.

Que les réalités prégnantes et tangibles se trouvent à l'échelon sub-régional.

- En effet, des associations et relations spécifiques, des entités douées d'une cohérence supérieure à celle des relations internationales "normales" existent bien:

Outre l'ASEAN déjà mentionnée, on trouve le continent nord-américain et la zone Asie-Pacifique.

- Le continent nord-américain connait des liens spécifiques entre le Canada, les Etats-Unis et dans une moindre mesure le Mexique (continuité géographique, mobilité des populations et travailleurs trans-frontaliers, accords de libre échange, etc.).

- De façon plus lâche mais néanmoins significative, la zone désignée en anglais par le terme "East Asia" et en français par "Asie-Pacifique" s'est de facto structurée à partir et autour du Japon, parallèlement au développement d'un processus d'"asianisation" (ou de "ré-asianisation"). Plusieurs révolutions industrielles se sont à l'origine déroulées dans cette zone avec succès, sur une échelle limitée mais significative.

Les conclusions d'Anne Androuais du Centre de recherches sur le Japon contemporain -CNRS - tendent à démontrer que des phénomènes d'entraînements économiques créés à partir du Japon y ont engendré des pôles de développement, selon un système économique hiérarchisé à trois niveaux inégaux : les nouveaux pays industrialisés asiatiques, l'ASEAN et la Chine. Les investissements directs japonais ont suivis là des logiques 
successives : politique de coopération économique pendant les années '60; modification de structure industrielle dans les années ' 70 ; internationalisation dans les années '80. "Le Japon a réussi à mettre en oeuvre une politique $d$ 'industrialisation régionale qui, non seulement lui a donné le plus grand avantage comparatif mais lui a aussi permis d'amorcer la construction économique de l'Asie". [Anne Androuais, "Les investissements japonais en Asie du Sud-Est : complèments ou concurrents de l'industrie au Japon", Revue d'Economie politique, 1985, n 3, pp. 320-345].

$\mathrm{D}^{\prime}$ une part, la cohérence de ce pôle est très supérieure à la cohérence de la zone Pacifique (ce qui n'est pas difficile).

D'autre part, cette cohérence est dans l'absolu d'un niveau moyen et historiquement déjà assez ancienne.

La nouveauté en l'occurrence réside donc dans le développement de l'intégration économique de ce pôle. Il faut néanmoins remarquer que ce pôle ne semble pas être une fin en soi, mais plutôt un tremplin vers l'économie mondiale (la dépendance d'un pays de la zone vis-à-vis de celle-ci étant d'autant plus forte que son économie est moins développée). Ces économies sont aujourd'hui impliquées dans l'économie mondiale.

L'attitude des "pays en place" à leur égard change $d^{\prime}$ ailleurs progressivement. Plusieurs études de l'OCDE notamment insistent aujourd'hui sur les désavantages à long terme (inhibition de la croissance, ...) des politiques défensives dirigées contre la pénétration des productions des Nics [OECD, "The Newly Industrialising Countries, Challenge and Opportunity, Paris, 1988 ]. Comme le souligne Achim von Heynitz, "political emphasis in the group of the major industrial countries has shifted toward 
the improvement of their sluggish reponse and inflexible adjustment. Thus, the four Asian Nies are seen increasingly as normal competitors within a broad array of competitive pressures rather that as a competitive threat". ["Discussion paper : some guidelines for an European policy toward the Asian newly industrializing economies", Feb. 1989, S.W.P., p. 12 ].

Une place doit leur être faite dans les structures institutionnelles économiques dominantes, ce qui impliquera des ajustements mais pas de bouleversements. (Un groupe de travail étudie d'ailleurs déjà les possibilités d'une coopération plus large avec les NICs).

\section{Derrière les fantasmes.}

En inversant la formule célèbre, on pourrait dire qu'il ne faut pas passer trop rapidement de l'adhésion à un mythe au refus de certaines réalités.

Les "théories du Pacifique" véhiculent beaucoup de généralisations abusives et de démonstrations biaisées, de fantasmes, de confusions d'entités et de points d'interrogation. Leur "Pacifique" est aujourd'hui, et très vraisemblablement demain encore, un mythe (hétérogénéité multi-dimensionnelle, absence de volonté commune, etc.).

La naissance d'une Communauté Pacifique ne peut être définitivement écartée, mais elle n'est concevable qu'à long terme et avec une probabilité très faible.

A la place du "nouveau centre du monde", de la "Communauté du Pacifique", du "siècle du Pacifique qui vient", 
nous voyons d'autres réalités dont certaines peuvent sembler évidentes mais qui sont occultées dans toutes ces théories.

- La première réalité est celle d'un monde où domine le clivage nations riches/reste du monde. La puissance, le "centre du monde" envisagé par les théories Pacifique passe par l'OCDE. La maîtrise de l'économie mondiale, la fixation des règles du jeu, la maîtrise technologique restent largement dans les mains de ses membres.

A ce niveau, l'appartenance à ce club est plus significative que l'appartenance à une entité géographique. Dans cette logique planétaire, les rapports de subordination /dépendance n'existent pas au bénéfice d'une zone sur les autres mais sont géographiquement disséminés.

- La deuxième réalité est celle d'une zone désignée par "bassin Pacifique" qui a retrouvé une certaine stabilité et dont une partie réunit les conditions favorables au développement économique, mais qui reste très éclatée. Globalement le bilatéralisme, qu'il soit politique ou économique, y domine toujours largement et le nationalisme est loin d'y être mort.

Il n'y a pas de "conflits d'allégeance". La spécificité nationale est encore vécue comme primordiale sinon exclusive (formulé autrement, le Japon est un pays japonais, les Etas-Unis sont un pays nord-américain; on ne peut les dénommer "pays pacifiquéens" comme on dirait de la Belgique ou de l'Italie qu'ils sont des pays européens).

- La troisième réalité est celle de l'émergence de régionalismes différenciés à une échelle qui n'est pas celle annoncée.

Derrière le concept de Pacifique, artificiel tant du point 
de vue politique, économique que géographique, on trouve plutôt l'addition d'une rive asiatique et d'une rive américaine, chacune poursuivant un régionalisme "soft". $\mathrm{Au}$ sein de ces deux zones se développent des cristallisations sub-régionales, des intégrations comparativement plus poussées.

- La quatrième réalité est celle de la sous-région Asie-Pacifique où est indéniablement localisé un pôle de croissance actuel, animé d'une dynamique propre.

- La cinquième réalité est celle des incertitudes planétaires et régionales qui pèsent sur les réalités qui précèdent.

Rien ne semble devoir troubler la stabilité régionale dont bénéficie dans l'ensemble le Pacifique.

Mais pour ne prendre qu'un des cas de figures, le probable désengagement partiel américain peut remettre cela en question. S'ill n'est pas en lui-même dangereux pour la stabilité régionale, il permet le retour d'incertitudes politico-militaires à un échellon subrégional. De vieux et nouveaux conflits nationaux, ethniques, religieux, ..., peuvent se développer, $s^{\prime}$ envenimer et avoir un effet de contagion qui ruinerait cet environnement favorable au développement économique. Moreau-Defarges décrit toujours l'Asie sous les mots de "hiérarchies partielles, inachevées et instables".

En envisageant un scenario moins catastrophiste mais qui $n^{\prime}$ en est que plus crédible, un krack boursier, des mesures protectionnistes en chaîne, le retrécissement des échanges mondiaux peuvent, par un jeu de dominos, précipiter la formation d'une réalité très différente : celle de deux 
intégrations économiques régionales marquées et introverties.

Autrement dit, pour utiliser la terminologie de Fernand Braudel, nous pourrions assister, à la place du siècle glorieux du Pacifique, à la formation de deux "économie monde" ("un morceau de la planète économiquement autonome, capable pour l'essentiel de se suffire à lui-même et auquel ses liaisons et ses échanges intérieurs confèrent une certaine unité organique"; un ensemble ayant ses limites, ses centres dominants, une hiérarchie entre zones sur et sous développées, mais transcendant frontières politiques et civilisations). ["Civilisation matérielle, économie et capitalisme", Tome III, Paris, 1979].

Comme l'écrit Achim Von Heynitz, "will the multilateral order finally collapse into a Pacific-American and an EuropeanMediterranean regional bloc?" [p. 34].

Voilà une question qui doit interpeller bien plus que celle $\mathrm{d}^{\prime}$ un hypothétique Pacifique! 


\section{BIBLIOGRAPHIE}

ANDROUAIS, Anne - "Les investissements japonais en Asie du SudEst : compléments ou concurrents de l'industrie au Japon", Revue d'économie politique, $\mathrm{n}^{\circ} 3,1985$, p. 320-345.

BLAUVELT, E. and DURLACHER, J. (eds) - "Sources of Asian/Pacific economic information", Hants, England : Gover, 1982, 2 vol.

BOGOMOLOV, A. - "La région du Pacifique : questions de coopération", La vie internationale, janvier 1987, Moscou, p. 33-40.

BUNGE F.M. \& COOKE M.W. (eds) - "Oceania - a regional study", 1985, US Gov., Wash. DC.

CALVOCORESSI, Peter - "World Politics since 1945", 5th ed., Longman, London, 1987, $546 \mathrm{p}$.

CHAPONNIERE Jean-Raphael - "La puce et le riz - croissance dans le Sud-Est asiatique", Armand Colin, Paris, 1985, 208 p.

CEPII - "Trois cas de relations Nord-Sud (Japon - Asie Pacifique; Brésil; OCDE; URSS - Afrique septentrionale)", n 4, Paris, s.d., 121 p.

CURRENT ECONOMIC INDICATORS - A quarterly statistical review of developments in the world economy, dpt. of Ec. and Soc. Aff, ONU.

ECONOMIC AND SOCIAL COMMISSION FOR ASIA AND THE PACIFIC - Quarterly Review, United Nations, Bangkok (Thailand).

ESCAP - "Annuaire général statistique", UN, Bangkok, 1988.

ESCAP - "Indicateurs statistiques", mars 1988, Bangkok.

GIBNEY, Frank G. - "The promise of the Pacific", BN/SR, vol. 32-1, n 1 , February 1988, USA, p. 90-96.

GOMANE J.P. et alii - "Le Pacifique Nouveau centre du Monde, Institut du Pacifique, Stratégies, Berger-Levrault, Paris, 1986, 363 p. 
GORDON, Bernard K. - "Politics and protectionism in the Pacific" - IISS, adelphi papers $\mathrm{n}^{\circ} 228$, London, spring $1988,82 \mathrm{p}$.

HARDING Harry (Ed.) - "China's foreign relations in the 1980's" - Yale Univ. Press, New Haven, 1984, 240 p.

HOLLAND, Harrison M. - "Managing diplomacy : the United States and Japan", Stanford, 1984.

HUNTLEY, James Robert - "Uniting the Democracies-Institutions of the emerging Atlantic/Pacific systems", NY 1980, 392 p.

JAPAN REVIEW OF INTERNATIONAL AFFAIRS - Japan Institut of International Affairs, vol. 1, 1987.

JOYAUX, François - "La nouvelle question de l'Extrême Orient", Paris 1985.

KIRBY, Stuart - "Towards the Pacific century - Economic development in the Pacific basin", special report $n^{\circ} 137$, The Economist Intelligence Unit, London, March 1983, 97 p.

KUMAO, Kaneko - "A new Pacific initiative : strengthening the PECC process", Japan review of international affairs, vol. $2 \mathrm{n}^{\circ} 1$, summer 1988, p. $67-90$.

LINDER, Staffan \& ROSTOW, W.W. - "The Pacific Challenge for world economic challenge", The American Economic Review, vol. 75, $\mathrm{n}^{\circ} 2$, May 1985, p. 279-291.

MILTON, Patton H. - "The Pacific basin : toward a regional future", Georgetown, KY : State Research Associates, 1980, 61 p.

de MONTBRIAL, Ph. (Ed.) - "RAMSES 1990 - système économique et stratégies", IFRI, Dunod, Paris, 1990.

MORISHIMA, Micho - "Capitalisme et confucianisme : l'éthique japonaise et la technologie occidentale, Paris, 1987. 
MOLEY, J. - "The Pacific basin - new challenges for the US" Academy of American political science, Proceedings, vol. 36, nº 1, 1986.

PROBLEMES POLITIQUES ET SOCIAUX - "L'avenir du Pacifique", Paris, $\mathrm{n}^{\circ} 498$, novembre 1984, p. 3-36.

RIEDEL, James - "Myths and reality of external constraints on development", Gover, Aldersshot (UK), 1987, 111 p.

SAUTTER, Christian - "Les dents du géant : le Japon à la conquête du monde", Paris, 1987.

SCALAPINO, Robert A. - "Regionalism in the Pacific : prospects and problems for the Pacific basin", The Atlantic Community Quarterly, vol. $26, \mathrm{n}^{\circ} 2$, summer 1988, p. 174-188.

SCHMIEGELOW, H. and M. - "Strategic pragmatism : Japanese lessons in the use of economic theory", New-York, Praeger publishers, 1989.

SERVOISE, René - "Le Pacifique - nouveau "nouveau monde"", Politique étrangère, Paris, $n^{\circ} 1$, printemps 1985, p. 101-118.

"South Pacific - an annotated bibliography on regional development" UNCRD project on regional dev. documentation, UN, 1984, 388 p.

VANDERMEERSCH, Léon - "Le nouveau monde sinisé", Perspectives internationales, PUF, Paris, 1986, 224 p.

YUJIRO, Eguchi - "Le Japon à l'ère du Pacifique", Cahiers du Japon, Tokyo, $\mathrm{n}^{\circ}$ 24, été 1985, p. 6-13. 